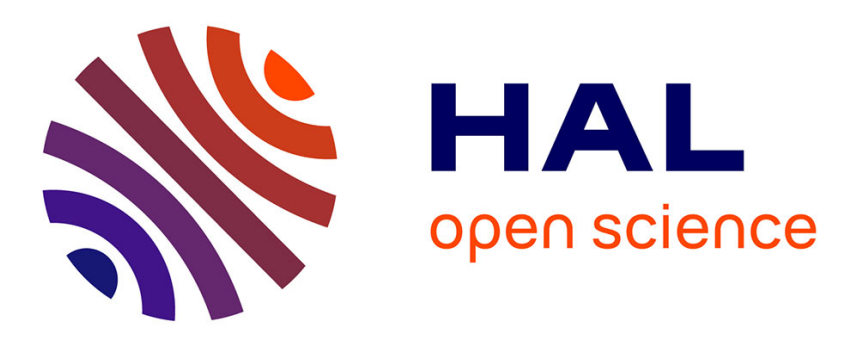

\title{
A Deep Multi-Task Learning Framework Coupling Semantic Segmentation and Fully Convolutional LSTM Networks for Urban Change Detection
}

\author{
Maria Papadomanolaki, Maria Vakalopoulou, Konstantinos Karantzalos
}

\section{- To cite this version:}

Maria Papadomanolaki, Maria Vakalopoulou, Konstantinos Karantzalos. A Deep Multi-Task Learning Framework Coupling Semantic Segmentation and Fully Convolutional LSTM Networks for Urban Change Detection. IEEE Transactions on Geoscience and Remote Sensing, 2021. hal-03140492

\author{
HAL Id: hal-03140492 \\ https://hal.inria.fr/hal-03140492
}

Submitted on 12 Feb 2021

HAL is a multi-disciplinary open access archive for the deposit and dissemination of scientific research documents, whether they are published or not. The documents may come from teaching and research institutions in France or abroad, or from public or private research centers.
L'archive ouverte pluridisciplinaire HAL, est destinée au dépôt et à la diffusion de documents scientifiques de niveau recherche, publiés ou non, émanant des établissements d'enseignement et de recherche français ou étrangers, des laboratoires publics ou privés. 


\title{
A Deep Multi-Task Learning Framework Coupling Semantic Segmentation and Fully Convolutional LSTM Networks for Urban Change Detection
}

\author{
Maria Papadomanolaki, Maria Vakalopoulou, and Konstantinos Karantzalos
}

\begin{abstract}
In this paper, we present a deep multi-task learning framework able to couple semantic segmentation and change detection using fully convolutional long short-term memory (LSTM) networks. In particular, we present a UNet-like architecture (LUNet) which models the temporal relationship of spatial feature representations using integrated fully convolutional LSTM blocks on top of every encoding level. In this way, the network is able to capture the temporal relationship of spatial feature vectors in all encoding levels without the need to downsample or flatten them, forming an end-to-end trainable framework. Moreover, we further enrich the L-UNet architecture with an additional decoding branch that performs semantic segmentation on the available semantic categories that are presented in the different input dates, forming a multi-task framework. Different loss quantities are also defined and combined together in a circular way to boost the overall performance. The developed methodology has been evaluated on three different datasets, i.e, the challenging bi-temporal high-resolution ONERA Satellite Change Detection (OSCD) Sentinel-2 dataset, the very high-resolution multitemporal dataset of the East Prefecture of Attica, Greece, and lastly, the multitemporal very high-resolution SpaceNet7 dataset. Promising quantitative and qualitative results demonstrated that the synergy among the tasks can boost up the achieved performances. In particular, the proposed multi-task framework contributed to a significant decrease of false positive detections, with $F 1$ rate outperforming other state of the art methods by at least $2.1 \%$ and $4.9 \%$ in the Attica VHR and SpaceNet7 dataset case respectively. Our models and code can be found at: https://github.com/mpapadomanolaki/multi-task-L-UNet
\end{abstract}

Index Terms-satellite, remote sensing, change detection, urban, deep learning, multi-temporal, Istms

\section{INTRODUCTION}

$\mathbf{T}$ HE diversity, volume and frequency of accessible satellite data has contributed decisively to numerous studies focusing on monitoring our environment based on multitemporal remote observations. Man-made and natural phenomena keep transforming the planet's structure, thus creating the need for effective monitoring methods. Urban growth is one of the most critical categories as the world's population keeps expanding in extremely fast rates occupying more and more of

M. Papadomanolaki is with the Remote Sensing Laboratory, National Technical University of Athens, Zographos, Greece as well as MICS Laboratory, CentraleSupélec, Université Paris-Saclay, Gif-sur-Yvette, France e-mail: (see mar.papadomanolaki@gmail.com).

M. Vakalopoulou is with MICS Laboratory, CentraleSupélec, Université Paris-Saclay, Gif-sur-Yvette, France as well as Inria Saclay, Gif-sur-Yvette, France.

K. Karantzalos is with the Remote Sensing Laboratory, National Technical University of Athens, Zographos, Greece.

Manuscript received XX XX, XX; revised XX XX, XX. the earth's surface. The continuous spread of both residential and commercial areas has resulted in several problems such as the diminishing of rural zones, the destruction of wildlife as well as increased levels of land, water and air contamination. For that reason, the systematic observation of urban sprawl, at high and very high spatial resolutions, becomes essential in order to fully comprehend future tendencies, take precautions and design more appropriate city infrastructures.

Indeed, identifying changes between satellite image pairs has been an active field of research for a very long time [1], [2], [3], [4] and for a wide variety of applications [5], [6], [7], [8]. At first, differences among remote sensing data were recognized mainly with manual, time-consuming approaches. Today, a diverse range of supervised and unsupervised change detection methods exist in the literature, like Markov Random Fields [9], [10], kernels [11], graphical models [12], [13] and Principal Component Analysis [14], [15]. Determining the exact timing of the change based on time-series data has also been an active field of research [16], [17], [18]. In addition, with the advances of machine learning during the last years, more and more techniques based on neural networks are emerging [19], [20], [21], [22], [23], [24], [25] aiming to create robust systems that can successfully tackle the change detection problem. Among the machine learning approaches, deep learning architectures are the ones that have captured most of the attention owing to state of the art on numerous computer vision applications [26], [27], [28], [29] including remote sensing [30], [31], [32], [33], [34], [35], [36], [37]. Even though successful results have been achieved on the remote sensing domain, the further development of deep neural networks is still hindered owing to insufficient datasets lacking multi-modal diverse information. Several supervised and unsupervised frameworks have been proposed, however the construction of robust networks is still an active research area especially for models that fully exploit multi-temporal information. Considering these, one can realize that much progress remains to be made until deep networks can account for fully operational and reliable tools for the remote sensing applications [38]. Despite these obstacles, research can still be conducted with the available resources, enriching the existing knowledge on several topics like semantic segmentation, change detection etc.

Change detection is in most cases associated to sequential data, making it necessary to evaluate temporal dynamics. Modeling the temporal relationship among features has been largely addressed by the computer vision community using 
Recurrent Neural Networks [39], [40] which have proven to be very powerful for a wide range of applications like tracking [41], action recognition [42], etc. Long Short-Term Memory Networks (LSTMs) [43] are also really effective for such problems [44], [45] since they moderate the vanishing gradient problem [46] when dealing with long-term dependencies. The combination of recurrent networks and deep learning architectures has also been adopted for time series tasks [47], [48], [49] in an attempt to produce more fruitful feature representations by extracting both spatial and temporal information during the learning process.

Recent remote sensing studies have considerably integrated deep learning techniques towards more effective change detection approaches. In [50], the authors propose a patch-based framework examining two different architectures (Siamese and Early Fusion) based on the ONERA Satellite Change Detection bi-temporal dataset. In the Siamese case, the two bi-temporal patches are processed by two distinct but identical branches of convolutional layers with shared weights. Then, the produced feature vectors are concatenated and fed to a series of fully connected layers. Regarding the Early Fusion case, the bi-temporal patch pairs are concatenated along the channel dimension before being passed as a single input to several convolutional and fully-connected layers.

In [51] the aformentioned network designs evolve into fully convolutional versions according to a U-Net like framework. More specifically, the fully convolutional Early Fusion (FCEF) structure downsamples the concatenated bi-temporal patch pair through the encoder, while the decoder upsamples it back to its original dimensions using also skip connections to enrich the feature attributes. For the fully convolutional siamese concatenation case (FC-Siam-Conc), the network is comprised of two separate encoding branches with shared weights that receive as input the bi-temporal pairs. In this approach, skip connections link the decoding steps with the concatenation of the two encoding parts' outputs. Lastly, the third proposed architecture also consists of two encoding branches only this time skip connections associate the decoding parts with the absolute difference of feature vectors that result from the corresponding encoding parts (FC-Siam-Diff).

In [52], a recurrent network $(\mathrm{ReCNN})$ is integrated into a convolutional architecture taking advantage of both spatial and temporal features under an end-to-end framework. Giving some more details, $5 \times 5$ patch pairs taken from corresponding pixels of bi-temporal images are processed by a succession of parallel but identical dilated convolutional layers. Next, the produced pair of feature vectors is passed through a recurrent neural network which calculates the temporal dependency between them. In the end, fully connected layers collect the temporal volume and decide if a change has occurred. Apart from change detection purposes, sequential satellite images have also been exploited for land cover classification as in [53], where multi-temporal Sentinel-2 agricultural parcels are transformed to unordered sets of pixels. Each set is passed through a pixel-set encoder resulting in a feature descriptor which is then processed by a temporal attention network [54]. Land cover classification purposes are also handled in [55] using convolutional recurrent layers which also mitigate the problem of cloud coverage [56].

Multi-task learning schemes [57], [58] have also been adopted when dealing with the change detection problem, since complementary assignments can provide the models with useful information during the training process, enhancing in this way the performances. In [59], urban change detection is coupled with the task of semantic segmentation on buildings using a fully convolutional siamese network, while a focal loss [60] is also utilized in order to ease the class imbalance problem. [61] also employs the multi-task learning approach, enhancing the architecture's ability to identify changes by performing simultaneously the task of land cover mapping. Here the optimal results are derived when the network is optimized in 2 phases; firstly the training process is focused on the identification of the different land cover semantic categories and secondly, the network is trained again for change detection using the land cover semantic segmentation weights as initialization. Finally, [62] combines multi-task learning with transfer learning to balance the distributions of labeled and non-labeled data. Specifically, an encoder-decoder network performs change detection on the bitemporal labeled input images, while the difference of unlabeled data is concurrently reconstructed by the network enriching the extracted features during the training procedure. After pretraining, finetuning methods are exploited for unsupervised training on the unlabeled data according to region-based and boundary-based strategies. Although fully convolutional models have resulted in very promising results regarding the semantic segmentation task [63], [64], [65], little effort has been made to adjust such frameworks for change detection related topics. Especially for LSTMs, the processing of multi-dimensional matrices remains a very challenging problem since in most cases satellite images need to be flattened in order to be imported to such networks.

In order to tackle the aforementioned challenges, we have designed, implemented and validated a deep multi-task learning framework able to couple semantic segmentation with fully convolutional long short-term memory (LSTM) networks for urban change detection applications. Regarding the fully convolutional LSTM structure, it has been designed by replacing the gating mechanisms with convolutional layers. Our main goal here was to combine spectral and spatial information, while taking advantage of the temporal relationship among the feature matrices avoiding the computationally expensive task of multiplying high dimensional feature vectors. The fully convolutional LSTM blocks are placed on top of each encoding level of a UNet-like deep architecture, capturing in this way temporal information for all the different resolution levels. This current study is an extension of our previous work [66] in which fully convolutional LSTMs were utilized to semantically segment the OSCD dataset. Here, the novel framework is further enriched by adding dropout layers to the hidden states of the LSTM blocks. In addition, an extra decoding branch is explored for the semantic segmentation of the available categories, providing the network with fruitful supplementary feature attributes during the training procedure. An ensemble of losses combined in a circular way is also employed for the optimization process. To sum up, this paper makes the following contributions: 
- A UNet-like architecture (L-UNet) is proposed which models the temporal relationship of spatial feature representations using integrated fully convolutional LSTM blocks on top of every encoding level. Each LSTM block operates on the given sequential input by defining the weights and biases of the gating mechanisms as convolutional layers, thus avoiding the multiplication of high dimensional matrices. In this way, the network is able to capture the temporal relationship of spatial feature vectors in all encoding levels without the need to downsample or flatten them, creating an end-to-end trainable framework.

- The L-UNet architecture has been further enriched with an additional decoding branch that performs semantic segmentation on the semantic categories that are presented in the different available input dates (multi-task L-UNet). Under this multi-task framework, different loss quantities are also defined and combined together in a circular way to boost the reported accuracy of the change detection task.

The rest of this paper is outlined as follows. Section II introduces the methodology along with the implementation details, the benchmark datasets and the employed quantitative metrics. Section III presents the experimental results and the qualitative and quantitative assessment, the comparison with the state of the art as well as the performance of the different components. Finally, section IV concludes this paper.

\section{MAterials AND MethodS}

\section{A. Recurrent Neural Networks}

Recurrent neural networks are commonly employed for problems which include time-dependent data as they are able to capture the temporal relationship among sequential features. In their simplest structure, such networks process data which come in the form of $X=\left[X_{1}, X_{2}, \ldots, X_{T}\right]$, where $X \in \mathbb{R}^{N}$ is a list containing information related to $t \in[1, \ldots, T]$ different time steps. In every time step $t$, the respective list element $X_{t}$ is multiplied element-wise with an associated weight matrix $W_{x}$. At the same time, a representation of previous list elements, also known as the 'hidden state', is multiplied with a weight matrix $W_{h}$. The sum of these quantities is then passed to a hyperbolic tangent function to produce the hidden state $H_{t}$ of the current time step. This chain process is described as

$$
H_{t}=\tanh \left(W_{x} \cdot X_{t}+W_{h} \cdot H_{t-1}\right),
$$

with $W_{x}$ and $W_{h}$ being shared across all time steps. It should be noted here that biases are omitted for convenience reasons. Since the weights are shared across all time steps, the structures of such a network are very much likely to suffer from the vanishing gradient problem [46]. More specifically, during backpropagation the contributions of each time step are summed up to the gradient, according to the chain rule of differentiation for composite functions. This results in a recursive derivative based on multiplicative dynamics that tends to zero if the gradients become very small or if there are several time steps [67]. Long Short-Term Memory Networks (LSTMs), firstly proposed by Hochreiter and Schmidhuber [43], mitigate this problem by introducing a memory cell, most commonly known as the 'cell state', which exploits gating functions in order to filter the flowing information more efficiently. Unlike conventional RNNs which employ a single hyperbolic tangent layer at each time step, LSTMs refine the input volumes by introducing four interrelated layers known also as gates. In particular, the additional operations involve the forget $(f)$ and input $(i)$ gates as follows

$$
\begin{aligned}
f_{t} & =\sigma\left(W_{f} \cdot\left(X_{t}, H_{t-1}\right)\right), \\
i_{t} & =\sigma\left(W_{i} \cdot\left(X_{t}, H_{t-1}\right)\right),
\end{aligned}
$$

where $\sigma$ is the sigmoid function while $W_{f}$ and $W_{i}$ are weight matrices employed for each gating unit. The forget gate employs a sigmoid function in order to throw away ineffectual feature representations while the input gate determines which information part is going to be utilized for the update of the cell state. Apart from $f$ and $i$ gates, every LSTM cell consists also of the cell gate $\left(c_{t}\right)$ and the output gate $\left(o_{t}\right)$ defined as

$$
\begin{gathered}
c_{t}=\tanh \left(W_{c} \cdot\left(X_{t}, H_{t-1}\right)\right), \\
o_{t}=\sigma\left(W_{o} \cdot\left(X_{t}, H_{t-1}\right)\right),
\end{gathered}
$$

where tanh is the hyperbolic tangent function while $W_{c}$ and $W_{o}$ are the corresponding weight matrices. The cell gate utilizes the hyperbolic tangent function to regulate the data and produce possible candidate cell state values, while the output gate further filters the information determining the outcome of the network. After that, the network is ready to create the new cell state by forgetting irrelevant features from the previous cell state and keeping valuable ones for the current cell state.

$$
C_{t}=f_{t} \cdot C_{t-1}+i_{t} \cdot c_{t},
$$

Finally, the cell state is given to a hyperbolic tangent function and it is also multiplied by the output gate result to produce the hidden state of current time step $t$.

$$
H_{t}=o_{t} \cdot \tanh \left(C_{t}\right),
$$

By meticulously filtering the flowing information through the gates, dependencies are maintained while the memory state is more properly conserved. Apart from that, back propagation

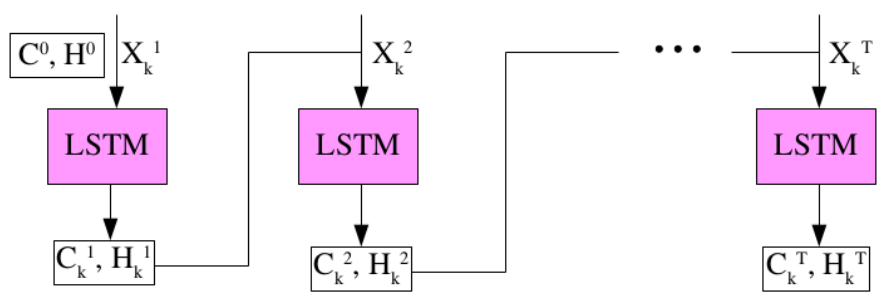

Fig. (1) The process based on which the temporal relationship among the spatial features is calculated. Cell state $C_{k}^{t}$, hidden state $H_{k}^{t}$ and input $X_{k}^{t}$ are given as input to the LSTM at each time step $t$ until the final hidden state $H_{k}^{T}$ is produced, where $T$ stands for the number of employed dates and $k$ for the encoding level. 
through time becomes more efficient since additive dynamics are integrated into the recursive multiplicative derivatives that take place when calculating the error gradients with regard to the recurrent weights. In this way, the vanishing gradient problem is less likely to appear.

\section{B. $L-U N e t$}

Even if the previous formulations capture successfully temporal relations on sequential datasets, they become a bit inefficient, augmenting significant the number of parameters, in the case of high dimensional data such as remote sensing images $I=\left[I_{1}, I_{2}, . ., I_{T}\right]$, where $I_{t} \in \mathbb{R}^{C h \times H h \times W d}$ with $C h$ denoting the number of spectral channels and $H h, W d$ the spatial dimensions of image $I_{t}$. In such a case we would end up with multiplications of immense multi-dimensional matrices, making the training process computationally expensive and hindering the model's convergence.

To deal with this predicament, weight matrices $W_{f}, W_{i}, W_{c}$ and $W_{o}$ have been replaced with single strided convolutional layers comprised of $3 \times 3$ kernels and padding equal to one. In the proposed UNet-like framework, temporal image volumes of $T$ different dates, each one in the form of $(B s \times C h \times$ $H h \times W d$ ), with $B s$ denoting the batchsize, are passed to the model. Each of the $I_{t}$ images is processed separately by the encoding layers using shared convolutional weights, with one LSTM network being placed on top of every encoding level. Figure 1 provides a graphical description on how the temporal relationship among spatial features is computed after each encoding level $k$, with cell state and hidden state being initialized as zero matrices of shape $(B s \times C h \times H h \times$ $W d$ ). Every illustrated box represents all the interior gating operations that take place inside the LSTM cell. By replacing the weight matrices with convolutional layers, each gating mechanism can now be defined as

$$
G_{k}^{t}=\Phi\left(W_{G_{k}^{t}} *\left(X_{k}^{t}, H_{k}^{t-1}\right)\right)
$$

where $G_{k}^{t}$ is the forget, input, output or cell gate at time step $t$ of encoding level $k, \Phi$ is an activation function and $W_{G_{k}^{t}}$ is a convolutional layer applied on the concatenation of the current input $X_{k}^{t}$ and the previous hidden state $H_{k}^{t-1}$ along the channel dimension. The filtered information outcome is then utilized for calculating the current cell state and hidden state using equations 1 and 2 . The final hidden state of every encoding level $k$ is collected in order to be later concatenated with the output of the respective decoding part. In this way the LSTM block acts as a skip connection for the L-UNet.

As far as optimization is concerned, we use a standard cross entropy loss.

$$
\operatorname{Loss}_{C E}=-\sum_{l=0}^{n} y_{s, l} \log \left(p_{s, l}\right)
$$

In the above expression, $n$ is the number of classes, $y_{s, l}$ is a binary indicator that shows if class $l$ is the correct answer for observation $s$, while $p_{s, l}$ holds the probability that observation $s$ belongs to class $l$. For the L-UNet case, the total loss for the optimization of the change detection task can be described as

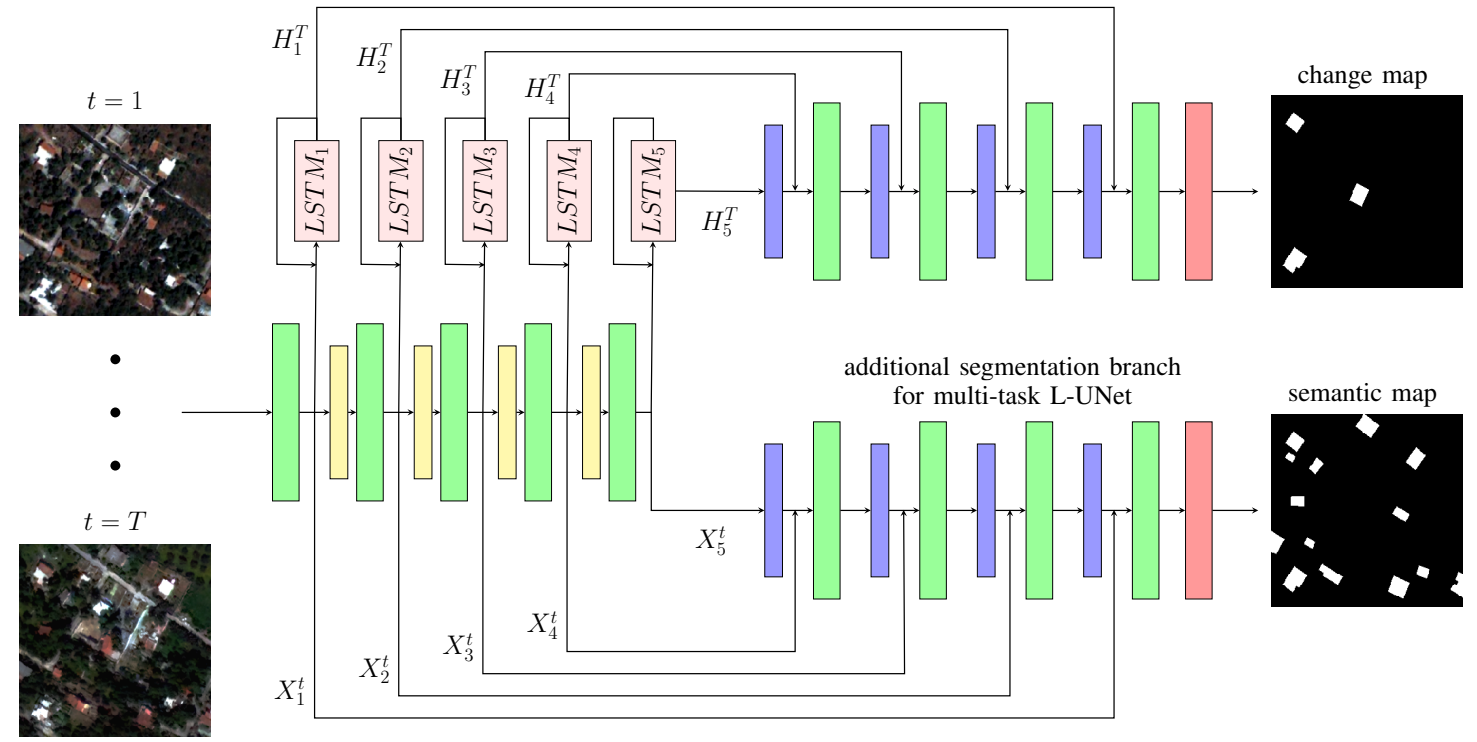

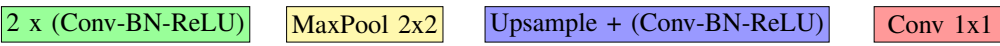

Fig. (2) The proposed multi-task L-UNet architecture consisting of 5 encoding levels. The upper decoding branch is responsible for the change detection task, concatenating the final hidden states of the corresponding LSTM blocks. The lower decoding branch performs semantic segmentation on the available semantic labels which for our experiments include the building footprints for the first and the last employed dates $(t=1$ and $t=T)$, each time concatenating the corresponding spatial feature vector. If the lower decoding branch is removed, then the architecture is considered a plain L-UNet as in [66]. 


$$
\operatorname{Loss}_{c h}=-\sum_{l=0}^{n} y_{s, l} \log \left(p_{c h(s, l)}\right)
$$

$p_{c h(s, l)}$ denotes the probability that observation $s$ belongs to class $l$, with $l$ indicating each of the available change semantic categories. Also, the variable $n$ here indicates the different types of change that may be available.

\section{Multi-task L-UNet}

Apart from calculating the temporal relationship among the data, features related to the semantic segmentation of the available categories can be also utilized by further customizing the proposed scheme with an auxiliary decoding branch. This branch performs semantic segmentation for the various input dates, with skip connections concatenating the spatial feature vectors of each encoding level. For our investigation, we chose to perform the training process using only the semantic maps of the first and the last date. This selection was based on the fact that the available change ground truth of the datasets describes the changes that have occured between the first and the last date. However, it is possible to use more dates or semantic categories depending on the application and the available computational resources. An overview of the proposed architecture is presented in Figure 2, summarizing the multitask learning framework. It should be noted here that in our experiments the semantic segmentation task is performed on two different categories (buildings / non-buildings) since these are the only available annotations that we have for the available dates. However, our method is modular and it can be adjusted to any number of available semantic and change categories, as our formulation is based on multiclass cross entropy loss for both segmentation and change detection tasks.

For the optimization of the proposed scheme we utilize an ensemble of loss quantities based on cross entropy, however any other kind of loss function can be employed. Five different loss entities are used in total during the training process, which are also combined together in a circular way in an attempt to reduce false positive detections. In particular, we use cross entropy loss $L_{0 s s_{c h}}$ as described in section II-B for the change detection task, as well as two more loss quantities for the building semantic maps of the first and the last available dates

$$
\operatorname{Loss}_{\text {seg }}^{t}=-\sum_{l=0}^{m} y_{s, l} \log \left(p_{\text {seg }(s, l)}^{t}\right)
$$

In the above equation, $t=\{1, \ldots, T\}$ and $p_{\text {seg }(s, l)}^{t}$ holds the probability that observation $s$ belongs to the $l$ semantic category for time $t$. In addition, cross entropy is employed for the definition of one more loss, Loss $s_{c h 2}$, that focuses on change detection by exploiting the features produced by the last convolutional layer of the semantic segmentation decoding branch. If we denote with $F_{\text {seg }}^{1}$ and $F_{\text {seg }}^{T}$ these features for the first and last date accordingly, then the features for the change detection can be defined as $F_{c h 2}=F_{\text {seg }}^{T}-F_{\text {seg }}^{1}$. In particular, we subtract the features of the first date from the features of the last date. This way, $\operatorname{Loss}_{\operatorname{ch} 2}$ corresponds to a cross entropy loss similar to equation 5 using the resulting $F_{c h 2}$ features. In the same manner, we calculate $\operatorname{Loss}_{\text {seg2 } 2}^{T}$ by combining the final features from the semantic segmentation and change detection branches as $F_{\text {seg } 2}^{T}=F_{\text {seg }}^{1}+F_{c h}$. That is, we add the features resulting from the semantic segmentation of the first date $\left(F_{\text {seg }}^{1}\right)$ with the features resulting from the last convolutional layer of the change detection decoding branch $\left(F_{c h}\right)$. As all these features are produced by the different branches, they fully exploit the representations that are produced by the multi-task L-UNet in a circular way. For the final optimization of the network we use the weighted sum of all these losses as

$$
\begin{array}{r}
\text { Total_Loss }=w_{1} \operatorname{Loss}_{c h}+w_{2} \operatorname{Loss}_{\text {seg }}^{1}+w_{3} \operatorname{Loss}_{\text {seg }}^{T} \\
+w_{4} \operatorname{Loss}_{\text {ch } 2}+w_{5} \operatorname{Loss}_{\text {seg } 2}^{T}
\end{array}
$$

where the sum of the weights $\left(w_{1}, w_{2}, w_{3}, w_{4}, w_{5}\right)$ is 1 .

\section{Datasets and Implementation Details}

The conducted experiments were based on three multispectral datasets; the high-resolution ONERA Satellite Change Detection (OSCD), the very high-resolution Attica VHR and the very high resolution SpaceNet7 dataset. We should highlight here that for all datasets, the change detection task is performed using 2 classes, namely change and no change. As a result, in equation 5, $n$ is equal to 2. For the Attica VHR and the SpaceNet7 datasets, the semantic segmentation task is performed for the first and the last available date using 2 classes, namely building and non-building. Hence, in equation $6, m=2$ and $t=\{1, T\}$. Further details for each of the datasets are provided in the next paragraphs.

1) ONERA Satellite Change Detection (OSCD): The OSCD dataset [50] consists of bitemporal Sentinel-2 satellite images depicting 24 different cities around the world. 13 spectral channels are available for each image pair while ground truth information is related to urban change and provided for 14 cities. Our setup follows the submission system guidelines ${ }^{1}$

\footnotetext{
${ }^{1}$ http://dase.grss-ieee.org
}

\begin{tabular}{cccccccc}
\hline Abudhabi & Beirut & Chongqing & Dubai & Hong Kong & Milano & Paris & Rio \\
\hline $2016 / 01 / 20$ & $2015 / 08 / 20$ & $2017 / 04 / 14$ & $2015 / 12 / 11$ & $2016 / 09 / 27$ & $2016 / 12 / 28$ & $2016 / 11 / 30$ & $2016 / 04 / 24$ \\
$2016 / 09 / 29$ & $2015 / 12 / 08$ & $2017 / 07 / 23$ & $2016 / 06 / 08$ & $2017 / 01 / 25$ & $2017 / 05 / 27$ & $2017 / 02 / 15$ & $2017 / 02 / 18$ \\
$2017 / 03 / 18$ & $2016 / 04 / 26$ & $2017 / 09 / 16$ & $2016 / 11 / 05$ & $2017 / 04 / 02$ & $2017 / 08 / 15$ & $2017 / 04 / 09$ & $2017 / 05 / 09$ \\
$2017 / 09 / 09$ & $2017 / 04 / 21$ & $2018 / 01 / 14$ & $2017 / 06 / 03$ & $2017 / 10 / 22$ & $2017 / 11 / 18$ & $2017 / 08 / 29$ & $2017 / 07 / 28$ \\
$2018 / 03 / 28$ & $2017 / 10 / 03$ & $2018 / 04 / 02$ & $2018 / 03 / 30$ & $2018 / 03 / 23$ & $2018 / 01 / 22$ & $2017 / 11 / 07$ & $2017 / 10 / 11$ \\
\hline
\end{tabular}

TABLE (I) To augment the bi-temporal information of the publicly available ONERA Satellite Change Detection (OSCD) dataset and go beyond image pairs, we collected and integrated three additional intermediate dates. In this Table the obtained dates are illustrated for some of the dataset's cities. From top to bottom, the first and last dates are the already provided ones by the OSCD dataset. 


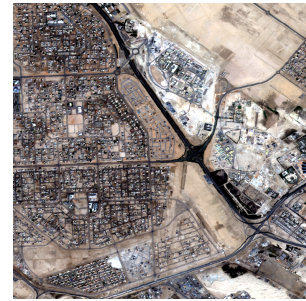

(a) $20 / 01 / 2016$

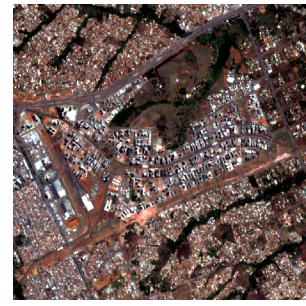

(e) $16 / 09 / 2015$

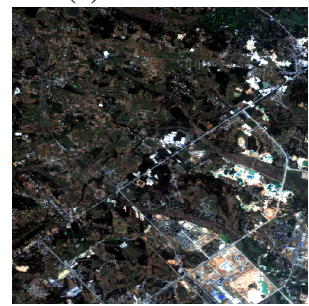

(i) $09 / 02 / 2016$

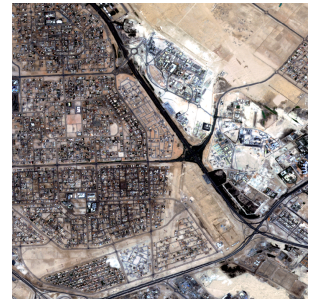

(b) $18 / 03 / 2017$

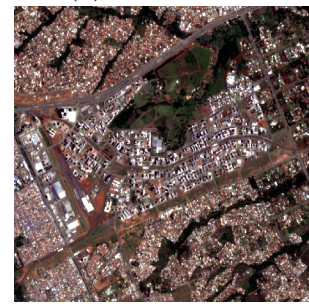

(f) $18 / 01 / 2017$

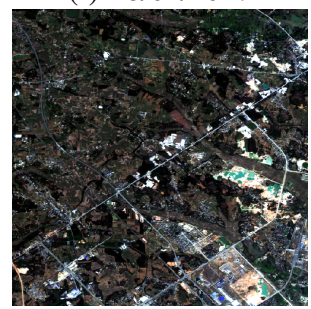

(j) $19 / 12 / 2017$

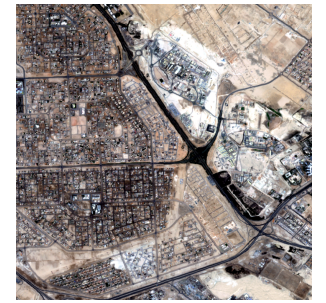

(c) $28 / 03 / 2018$

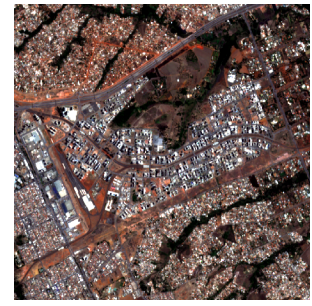

(g) $15 / 10 / 2017$

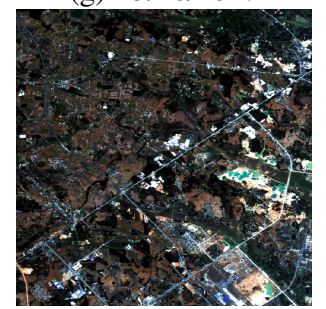

(k) $09 / 03 / 2018$

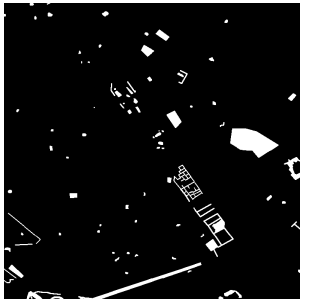

(d) Ground truth

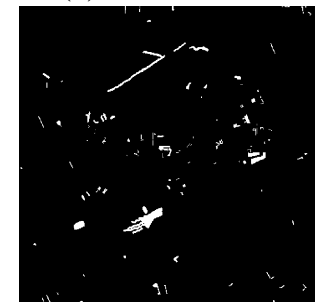

(h) Ground truth

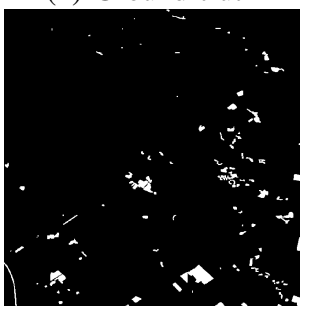

(1) Ground truth

Fig. (3) Indicative images from the OSCD training dataset for three different dates (in the form of dd/mm/yyyy) along with the corresponding change ground truth. From top to bottom, the illustrated cities are: Abu Dhabi, Aguasclaras, Beihai.

where the 14 image pairs are used for training and the rest for testing. We further enriched the OSCD dataset with additional Sentinel-2 images depicting the provided cities in different times. The additional dates include Sentinel-2 images captured between the provided bitemporal dates, adapting them as much as possible to cover different periods of the season. In Table I we present the timestamps of Sentinel-2 images used for 8 different cities. It should be mentioned here that all extra images were coregistered according to the provided OSCD bi-temporal dataset. In Figure 3 a part of the training images is illustrated for three different cities. The percentage of the change pixels for the OSCD collection constitute only the $2.3 \%$ of the training dataset.

As far as the training process is concerned, patches of size $32 \times 32$ were extracted with a stride of either 6 in case change pixels were included, or 32 in case no change pixels were contained exclusively. This strategy was applied as a stratified sampling approach to enrich the training samples that involve change features. In addition, more data augmentation techniques mainly used by the computer vision community, namely flipping in all possible angles proportional to 90 degrees, were implemented for patches whose number of change pixels exceeded the threshold of 5\% for the entire patch. A total of approximately 32000 patches containing both change and no change pixels resulted from the 14 training cities while 8000 were intended for validation purposes. It should be mentioned here that for the experiments we utilized the 4 high resolution channels of Sentinel-2 satellite; Red,
Green, Blue and Near-InfraRed. Finally, this dataset was used to evaluate the L-UNet architecture alone, since semantic annotations are available only for the change samples. Thus, with no semantic annotations for the different available dates, the multi-task L-UNet can not be implemented.

In order to take advantage of the entire dataset, our final predictions were produced by an ensemble of different trained models following a cross-validation scheme. Giving some more details, the training patches were divided into five equal parts and the same model was optimized five times using all possible combinations of the training dataset partitions. Then, predictions for the testing images were produced from all five models, with the final result being formulated by averaging the five model outcomes. It should be mentioned here that since the testing ground truth is not publicly available for this dataset, the quantitative results are obtained by submitting our predictions online ${ }^{1}$.

2) Attica VHR: This dataset includes 5 multispectral very high-resolution (VHR) images illustrating a $9 \mathrm{~km}^{2}$ region in the East Prefecture of Attica, Greece. All images were acquired by Quickbird and WorldView-2 between the years of 2006 and 2011. Specifically, the images of 2006 and 2007 were captured by Quickbird, while images of 2009, 2010, and 2011 were captured by WorldView2 satellite. Every sample is pansharpened and atmospherically corrected, while the ground truth has been manually annotated by remote sensing experts after an attentive and time-demanding photo-interpretation. For this dataset, ground truth is provided for the building 


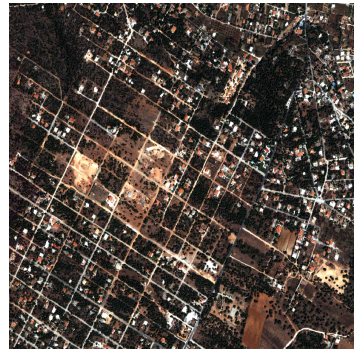

(a) 2006

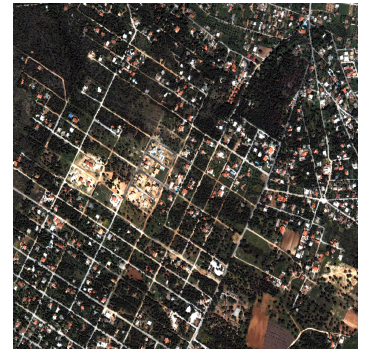

(b) 2009

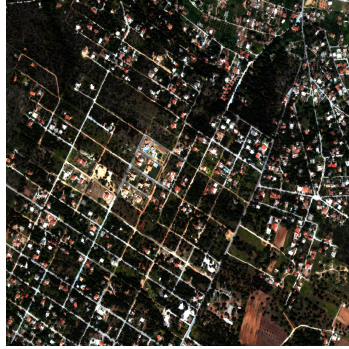

(c) 2011

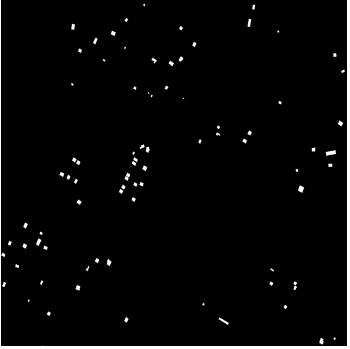

(d) Ground truth

Fig. (4) Training data from the Attica VHR dataset for three different years along with the corresponding change ground truth.

changes as well as for the building footprints of every single available date. It should be mentioned here that since we have two different sensors, Quickbird images were resized to the WorldView2 resolution which is approximately 8000 by 7000 pixels. In Figure 4 a training area for three different years along with the corresponding change ground truth is presented.

The whole region was divided into 36 equal nonoverlapping subregions of approximate size 1100 by 1300 pixels; 28 of them were used for training, 4 for validation and 4 for testing. For the training process, patches of size $64 \times 64$ were produced with a stride of either 32 in case change pixels were included, or 64 in case the patch did not include any change pixels at all. This strategy was applied as a data augmentation approach to enrich the change semantic category since it is extremely scarce compared to the no change one. Specifically, the percentage of change pixels in relation to the whole dataset is only $1.2 \%$. In addition, patches whose number of change pixels exceeded the threshold of 3\% were randomly flipped in all possible angles proportional to 90 degrees while their brightness, contrast and saturation levels were also randomly altered. Approximately 20200 patches containing both change and no change pixels were back propagated through the models for training, while 4000 were employed for validation.

3) SpaceNet7: This dataset was recently released for multitemporal building detection in one of NeurIPS 2020 challenges. It consists of multi-temporal satellite image cubes at 4 meter resolution, illustrating regions from all six continents of the earth. Red, Green, Blue and Near-Infra-Red are the available spectral channels, while each image is approximately 1024 by 1024 pixels. Each region is depicted at different months spanning across the years of 2018, 2019 and 2020, with the largest data cube containing 24 dates. 60 image cubes are intended for training, while 20 of them are used as test and they are evaluated through an online submission process. The ground truth for this dataset includes building footprints for each of the available dates, with an aim to monitor urban development. Competition participants are supposed to track the building locations for all the different dates, showing in this way the urban extension for each region.

In order to provide an additional evaluation benchmark for this work, we employed the 60 available training image cubes splitting them in 40 items for training, 10 items for validation and 10 items for testing. We produced the change ground truth by subtracting the building footprints of the first date from the building footprints of the last date. In Figure 5 one can observe a training sample from the SpaceNet7 dataset for four different dates. It should be mentioned here that SpaceNet7 contains additional preprocessed versions of the available RGB-NIR images where the clouds have been masked. For our investigation however we used the raw images so that the explored models can be evaluated on real conditions. In our experiments, the time-series related methods were examined using 10 dates. Patches of size $32 \times 32$ were produced with a stride of either 16 in case change pixels were included, or 32 in case the patch did not include any change pixels at all. Approximately 56000 patches were produced and intended for training purposes while 10000 were used for validation. Similarly to the previous datasets, change and no change samples are again extremely uneven, with change samples making up only $0.94 \%$ of the whole dataset.

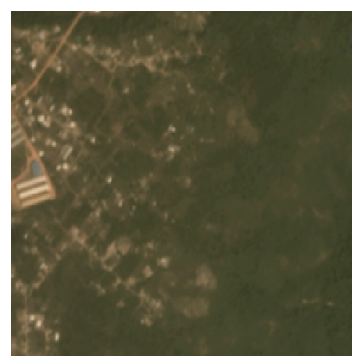

(a) $01 / 2018$

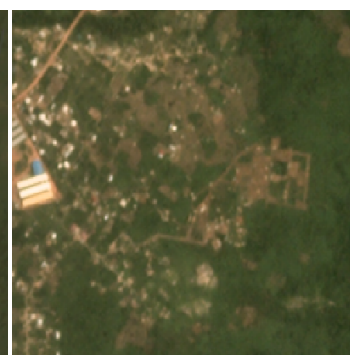

(b) $04 / 2018$

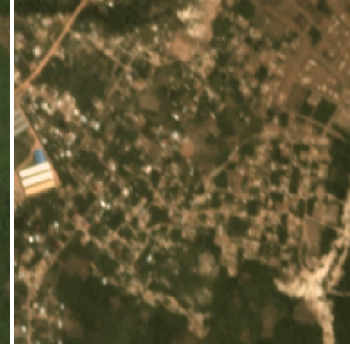

(c) $04 / 2019$

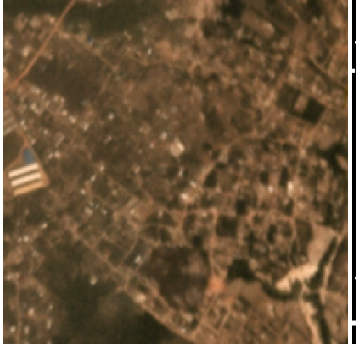

(d) $01 / 2020$

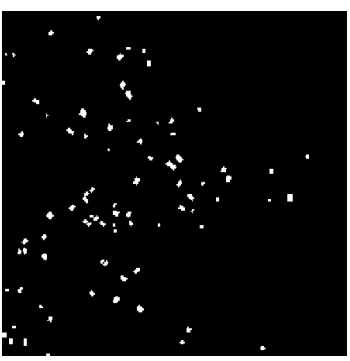

(e) Ground truth

Fig. (5) Training data from the SpaceNet7 dataset for four different dates along with the corresponding change ground truth. 


\section{E. Optimization}

Hyperparameters were similar for both datasets, picking Adam optimizer with a learning rate of $10^{-4}$. Batchsize was 32 for the OSCD dataset, 10 for the Attica VHR dataset and 2 for the SpaceNet7 dataset. Early stopping criteria were employed for every adopted approach in order to cease the training process and pick the optimal network weights. All applied methods needed less than 60 epochs to converge, while all experiments were implemented using the PyTorch deep learning library [68] on a single NVIDIA GeForce GTX TITAN with 12 GB of GPU memory. Each class was associated with a weight inversely proportional to the total pixel number included in it for the cross entropy loss. Furthermore, for the evaluation of multi-task L-UNet, a grid search was employed to determine the weight values for equation 7. Specifically, regarding the Attica VHR dataset $w_{1}$ was equal to 0.6 and the rest of the weights equal to 0.1 . In the SpaceNet7 case, $w_{1}$ was equal to 0.8 while the rest of the weights were equal to 0.05 . For the OSCD dataset, as we have already mentioned we evaluated only L-UNet since there is no available ground truth for the semantic categories of the different dates. Hence, $w 1$ was equal to 1 while the rest of the weights were equal to 0 . Lastly, it should be clarified here that the employed ground truth in each dataset case is related to the changes that have occured between the first and the last date.

\section{F. Quantitative Evaluation Metrics}

To assess the quality of the results we employed five different evaluation metrics: Precision, Recall, F1 score and Balanced Accuracy. They can be derived from the calculated TP (True Positives), FP (False Positives), TN (True Negatives) and FN (False Negatives) values. If we have observations belonging in $l$ different categories, then TP is the number of observations that have been correctly classified as $l$. FP is the number of observations that have been wrongly classified as $l . \mathrm{TN}$ is the number of observations that have been rightly recognized as not belonging to $l$. Finally, FN represents the observations that belong to $l$ but the model has associated them to another category.

$$
\begin{gathered}
\text { Precision }=\frac{T P}{T P+F P} \quad \text { Recall }=\frac{T P}{T P+F N} \\
F 1=\frac{2 \cdot \text { Precision } \cdot \text { Recall }}{\text { Precision }+ \text { Recall }} \\
\text { Balanced_Accuracy }=\frac{\frac{T P}{T P+F N}+\frac{T N}{T N+F P}}{2}
\end{gathered}
$$

\section{EXPERIMENTAl RESUlts AND Discussion}

In this section we evaluate each of the components of the proposed formulation benchmarking their performance. Moreover, we provide comparison with state of the art deep learning-based methods for change detection.

\section{A. Comparison with state-of-the-art methods}

Experiments were conducted using various number of dates, while the results were compared with fully convolutional networks [51], multi-task learning methods described in [59] and [61] as well as methods proposed for time-series datasets [55], [69]. All these literature methods were adjusted, using the same backbone U-Net-like architecture to ensure reliable comparison. In the following subsections, details are given for every investigated framework.

1) Method in [59]: Based on a UNet-like architecture, change detection and building semantic segmentation were performed simultaneously on the input dates, using two different decoding branches. In addition, two separate loss quantities were employed; cross entropy for the building semantic maps of the first and last date as well as the focal loss [60] for the change detection task.

2) Method in [61]: For the method in [61], we firstly trained the plain UNet-like model on the building semantic segmentation task using the first and last available dates. Then, using these weights as initialization, we trained the network once again on the change detection task, using as skip connections the absolute difference of the produced encoding feature vectors.

3) FC-Siam-Conc: In the FC Siamese Concatenation case [51] (FC-Siam-Conc), the encoder was comprised of several encoding streams depending on the number of processed dates. The streams had shared weights and identical configuration, with all the feature vectors produced after each encoding step being concatenated with the feature vector of the respective decoding step.

4) FC-EF: As far as the FC Early Fusion [51] (FC-EF) approach is concerned, the different temporal input volumes were concatenated along the channel dimension before being passed through the network. Skip connections concatenated the encoding outputs with the corresponding decoding ones.

5) FC-Siam-Diff: The FC Siamese Difference method [51] (FC-Siam-Diff) followed the same principles as FC-SiamConc, although this time the concatenation of skip connections was performed using the absolute difference of the resulting encoding feature volumes.

We should mention here that FC-Siam-Conc and FC-SiamDiff were also evaluated in a multi-task setting, by adding a supplementary decoding branch for the semantic segmentation task. In the FC-EF case, semantic segmentation could not be performed simultaneously with change detection since the different dates are fused along the channel dimension before being passed through the model, preventing in this way the construction of separate spatial feature vectors for each individual date.

6) LSTM [55]: In this work, sequential recurrent encoders were employed to perform the task of land cover classification based on image time-series. Specifically, the multi-temporal volume was given as input to a convolutional layer, the output of which was split into four equal parts, representing the four different gates of the LSTM structure. This forward pass was implemented in a bidirectional way, namely the input dates were passed to the encoder in sequential and reverse order. The 
final cell states were concatenated and transformed to softmaxnormalized activations so that the prediction map could be produced.

7) Method in [69]: Finally, the authors here have proposed a network for crop type classification from multi-temporal data. Specifically, the sequential input was passed through a succession of convolutional layers which downsample and upsample it back using also skip connections. The produced feature vector was then given to a convolutional LSTM so that the temporal relationship between the dates could be computed.

\section{B. Evaluation of the L-UNet}

In this section, we evaluate the use of fully convolutional LSTMs, integrated as skip connections on the UNet-based architecture. To benchmark their performance we report results on the three outlined datasets and we compare them with other change detection approaches and in particular methods proposed in [51], [55] and [69].

Looking at the results on the OSCD dataset (Table II), one can observe that L-UNet and FC-EF methods result in higher precision rates as additional temporal information is integrated, demonstrating their important contribution on the lessening of false positives. On the other hand, recall rates increase only in the FC-Siam-Diff case which means that even though in most of the methods the number of false positive detections is ameliorated, false negative pixels continue to exist in a large quantity. L-UNet produces the best precision and F1

\begin{tabular}{lllllll}
\hline Models & Dates & Precision & Recall & F1 & BA & Time(sec) \\
\hline \multirow{2}{*}{ FC-Siam-Conc [51] } & 2 & 59.32 & 54.73 & 56.93 & 76.34 & $\approx 4$ \\
& 5 & 62.51 & 50.12 & 55.63 & 74.24 & $\approx 4$ \\
& 60.45 & 50.46 & 55.01 & 74.33 & $\approx 5$ \\
FC-EF [51] & 2 & 45.46 & $\mathbf{7 1 . 4 2}$ & 55.56 & $\mathbf{8 3 . 3 7}$ & $\approx 3$ \\
& 3 & 53.05 & 59.74 & 56.20 & 78.43 & $\approx 4$ \\
& 5 & 59.09 & 55.56 & 57.27 & 76.73 & $\approx 5$ \\
\multirow{2}{*}{ FC-Siam-Diff [51] } & 2 & 60.86 & 54.07 & 57.26 & 76.09 & $\approx 3$ \\
& 5 & 57.13 & 50.63 & 53.68 & 74.28 & $\approx 4$ \\
LSTM [55] & 5 & 45.04 & 57.92 & 56.44 & 77.67 & $\approx 5$ \\
Method in [69] & 5 & 62.62 & 54.24 & 49.22 & 75.32 & $\approx 5$ \\
\hline \multirow{2}{*}{ Ours (L-UNet) } & 2 & 54.29 & 69.35 & 55.20 & 73.87 & $\approx 7$ \\
& 3 & 63.49 & 55.01 & 58.34 & 80.08 & $\approx 4$ \\
& 5 & $\mathbf{6 4 . 4 2}$ & 53.09 & 58.21 & 75.75 & $\approx 5$
\end{tabular}

TABLE (II) Quantitative evaluation of the proposed L-UNet on the testing part of the OSCD dataset. Precision, recall and F1 rates are associated to the change class, while Balanced Accuracy (BA) is also provided. All the rows demonstate results using the RGB-NIR bands with the last column indicating the time needed by each method to produce annotations for a testing image of dimensions $550 \times 550$.
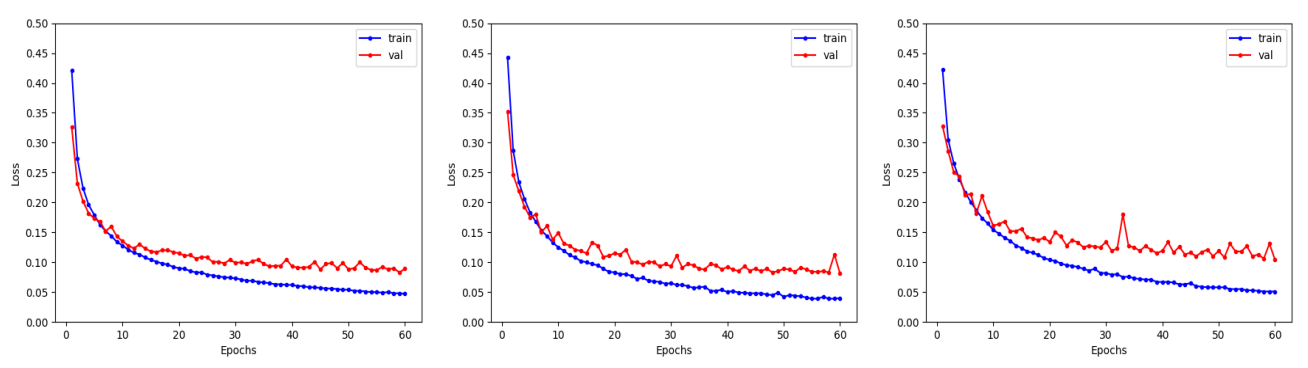

Fig. (6) Training and validation loss curves for different models trained with 5 dates on the OSCD dataset. From left to right: FC-SiamConc, FC-EF, FC-Siam-Diff, proposed L-UNet.

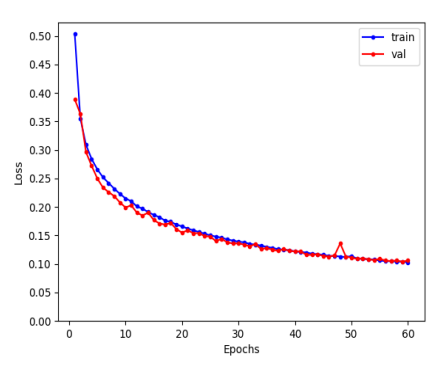

scores, meaning that the total number of false negative and false positive change pixels is smaller compared to the rest of the methods. The FC-EF bitemporal approach attains the highest recall and balanced accuracy metrics when employing 2 dates. However, in this case the precision rate is lower than $50 \%$ indicating a high number of false positive values, which is one of the main problems in change detection. In addition, the highest F1 score for FC-EF is attained in the case of 5 dates and it is approximately $1.7 \%$ lower than L-UNet. As far as time-series approaches are concerned, the method proposed in [69] produces a higher F1 score compared to the LSTM case [55]. Both approaches however result in lower accuracy metrics compared to the rest of the methods, except the method presented in [69] which attains the second best precision score, after L-UNet.

Moreover, training and validation curves are presented in Figure 6 for some of the investigated approaches using 5 dates. All the evaluated methods converge, however, the training of the proposed model seems to be smoother and more stable without very high variations between the training and validation performances.

We should mention here that since the ground truth for the testing images is not publicly available, we could not provide a thorough qualitative evaluation with proper illustration of TP, FP and FN regions. Nevertheless, in Figure 7 some advantages of the proposed L-UNet can be observed for the methods that attained the highest F1 scores according to Table II. In the first row there is an example where no change has taken place between the different dates, however all compared methods get disorientated by the existing cloud. In the case of L-UNet we can see that even though false positive pixels exist, they are less than the rest of the approaches. The proposed method also seems to be robust on changes that are not related to urbanization, reducing false positive detections. Specifically, in the second row our formulation is the only one that does not highlight agricultural changes as changed areas. Finally, in the third row we can observe that L-UNet has detected successfully urban changes, reporting less false positives (regions indicated with red circles).

Additional experiments on the Attica VHR dataset for the change detection task are presented in Table III. One can observe that the highest recall and balanced accuracy rates have been achieved by the LSTM [55] while the best precision and F1 scores have been attained by the L-UNet approach. In the LSTM [55] case however, the precision rate is 


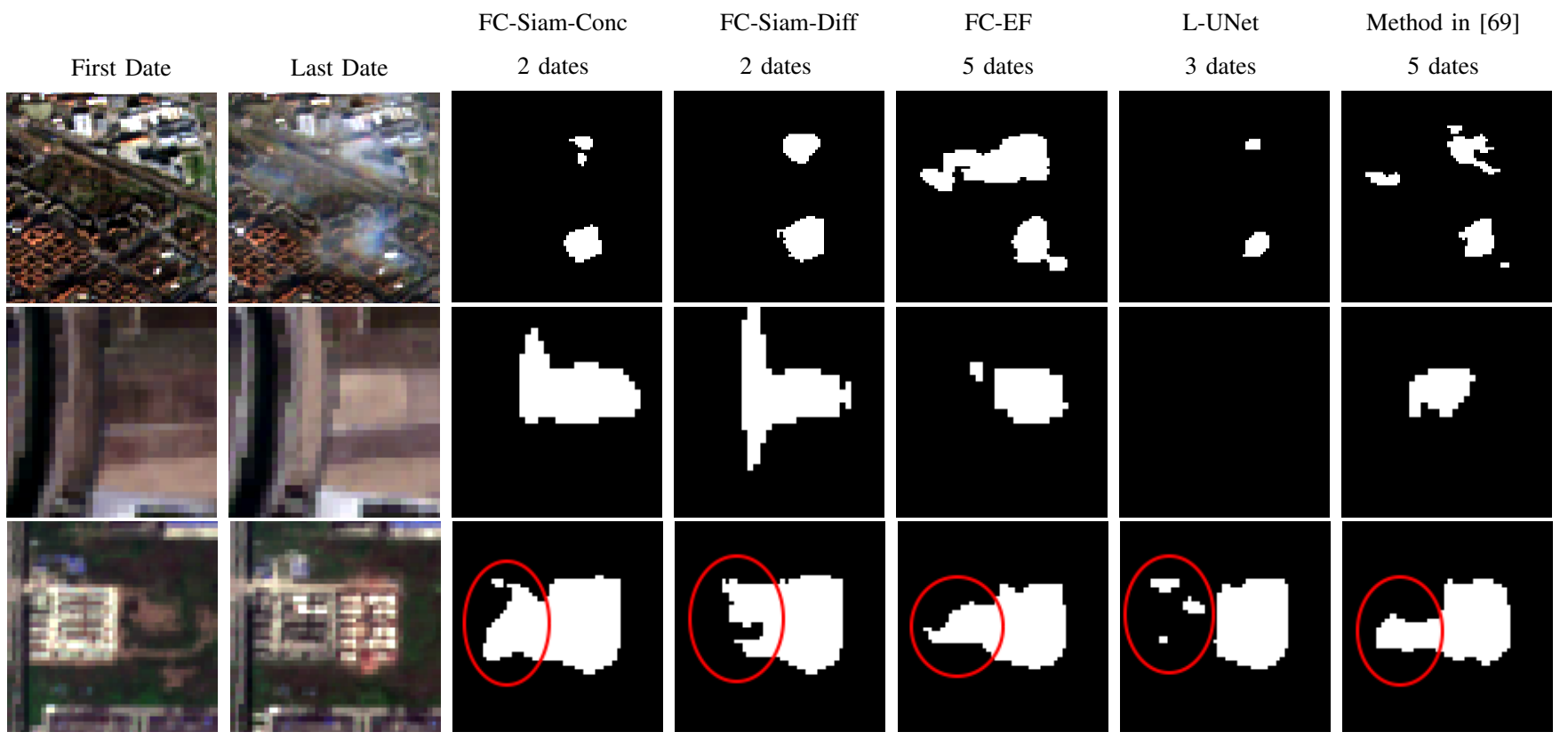

Fig. (7) Qualitative evaluation of the proposed L-UNet on zoomed testing regions of the OSCD dataset. 1st column: RGB images of the first available date, 2nd column: RGB images of the last available date, 3rd column: FC-Siam-Conc with 2 dates, 4th column: FC-Siam-Diff with 2 dates, 5th column: FC-EF with 5 dates, 6th column: L-UNet with 3 dates, 7th column: Method in [69] with 5 dates.

\begin{tabular}{lllllll}
\hline Models & Dates & Precision & Recall & F1 & BA & Time(sec) \\
\hline \multirow{2}{*}{ FC-Siam-Conc [51] } & 2 & 42.47 & 56.52 & 48.49 & 78.00 & $\approx 13$ \\
& 5 & 43.24 & 56.94 & 49.15 & 78.21 & $\approx 14$ \\
& 5 & 46.62 & 59.03 & 52.09 & 79.28 & $\approx 16$ \\
FC-EF [51] & 2 & 41.10 & 55.53 & 47.24 & 77.49 & $\approx 13$ \\
& 5 & 45.50 & 52.32 & 48.67 & 75.94 & $\approx 14$ \\
& 5 & 43.85 & 52.95 & 47.97 & 76.24 & $\approx 15$ \\
FC-Siam-Diff [51] & 2 & 45.67 & 56.80 & 50.63 & 78.17 & $\approx 13$ \\
& 5 & 46.90 & 54.18 & 50.28 & 76.88 & $\approx 14$ \\
LSTM [55] & 5 & 31.45 & 40.59 & 41.02 & 70.10 & $\approx 15$ \\
Method in [69] & 5 & 40.63 & 61.22 & 48.84 & 80.31 & $\approx 20$ \\
\multirow{2}{*}{ Ours $($ L-UNet) } & 2 & 47.25 & 55.21 & 50.92 & 77.39 & $\approx 14$ \\
& 3 & 43.15 & 61.08 & 50.57 & 80.26 & $\approx 15$ \\
& 5 & $\mathbf{4 7 . 9 6}$ & 60.19 & $\mathbf{5 3 . 3 8}$ & 79.87 & $\approx 17$ \\
\hline
\end{tabular}

TABLE (III) Quantitative evaluation of the proposed L-UNet on the testing part of Attica VHR dataset. Precision, recall and F1 rates are associated to the change class, while Balanced Accuracy (BA) is also provided. All the rows demonstrate results using the RGBNIR bands with the last column indicating the time needed by each method to produce annotations for a testing image of dimensions $1200 \times 1300$

exceptionally low, which means that false positive predictions have dramatically rized. The much higher F1 score in the LUNet case demonstrates the finer balance of false positive and false negative predictions, especially with the integration of more temporal information. The FC-Siam-Conc model is also boosted when additional dates are employed. FC-EF reports poor results, especially if we consider that precision rates remain always below $46 \%$ revealing the existence of many false positive detections. Lastly, in the FC-Siam-Diff case, even though F1 score reaches approximately the rate of 50\%, low precision levels reveal the large number of false positives.

In Figure 8, some qualitative outcomes are illustrated on zoomed regions from the Attica VHR testing areas. Identifying changes on buildings without additional information for the building class is quite challenging and can be sensitive to illumination changes. In the first row, one can notice that only L-UNet with 5 dates identifies that the depicting images do not contain any change related to the building class. Rooftop alterations in the second row have disoriented FC-Siam-Diff with 2 dates, FC-EF with 5 dates and the method in [69], whereas FC-Siam-Conc with 5 dates and L-UNet with 5 dates have addressed them successfully. Continuing with the third row, we can observe that the proposed approach has correctly identified that the swimming pool does not correspond to a building change category, in contrast to the rest of the methods. The total number of false negative and false positive pixels seems to be lower for L-UNet in the fourth row, while in the fifth row, the most successful detections have been attained by FC-Siam-Conc with 5 dates and L-UNet with 5 dates. In general, L-UNet and the addition of the LSTMs on the different encoding levels, seem to capture semantic changes in a better way.

As far as inference time is concerned, we can observe that L-UNet does not require more time to produce annotations, compared with the rest of the methods.

\section{Evaluation of the multi-task L-UNet}

In Table IV, we provide a comparison of the proposed multitask L-UNet with state of the art change detection methods. In particular, we compare our architecture with the models in [51] transformed into a multi-task setting, as well as the multitask methods in [61] and [59]. On the left part of Table IV, the evaluation of the change detection task is demonstrated, while on the right part, different metrics for the building semantic segmentation are provided.

Starting with the methods described in [51], compared to Table III one can observe that in the FC-Siam-Conc approach, the integration of building semantic segmentation ameliorates 


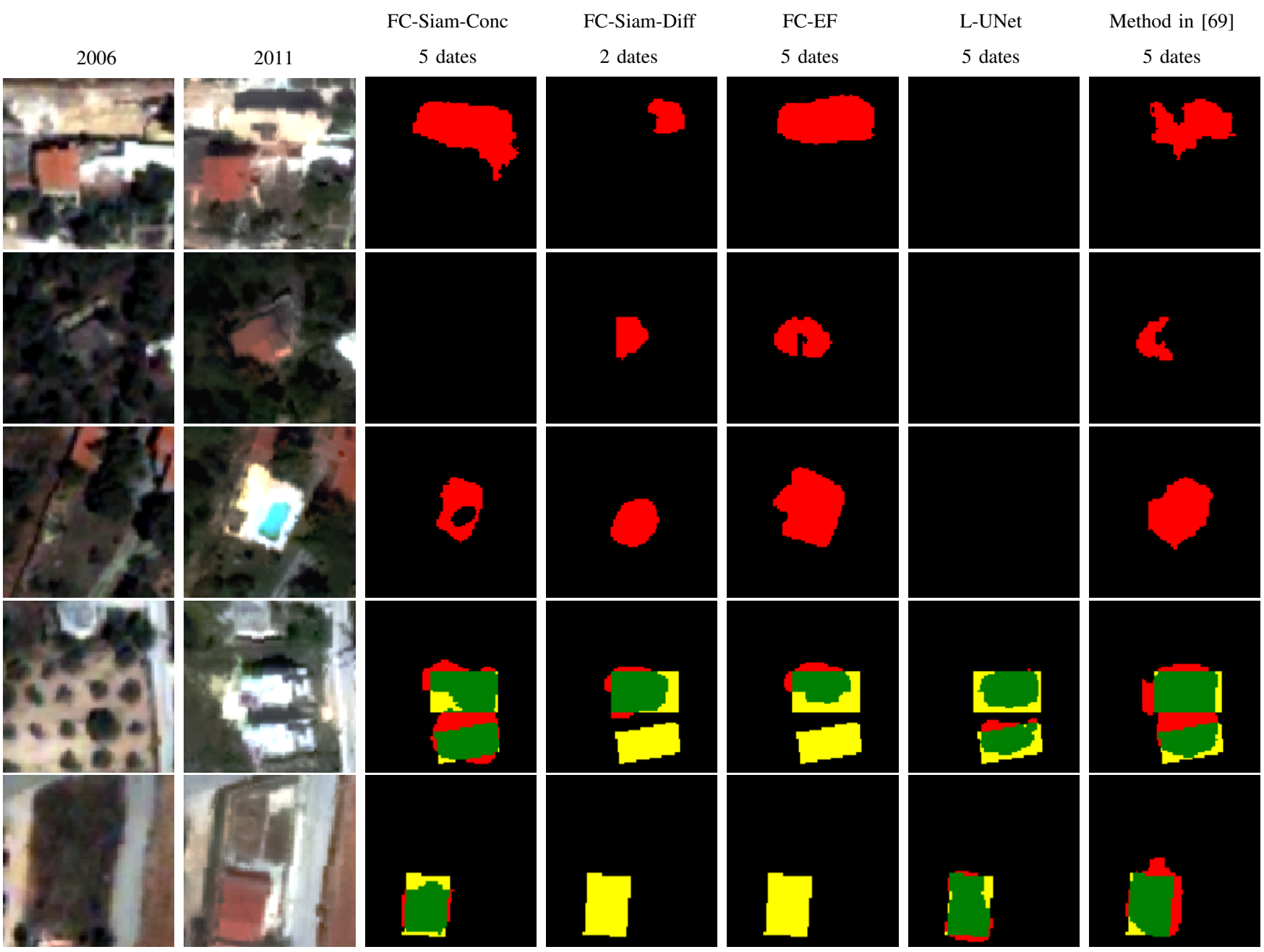

Fig. (8) Qualitative evaluation of the proposed L-UNet on zoomed regions of the Attica VHR testing areas for the change detection task. 1st column: RGB images of 2006, 2nd column: RGB images of 2011, 3rd column: FC-Siam-Conc with 5 dates, 4th column: FC-Siam Diff with 2 dates, 5th column: FC-EF with 5 dates, 6th column: L-UNet with 5 dates, 7th column: Method in [69] with 5 dates [Green: True Positives, Black: True Negatives, Red: False Positives, Yellow: False Negatives]

the F1 score especially in the case of 5 dates where the precision rate also rises above $50 \%$. Regarding FC-Siam-Diff, although recall rate and balanced accuracy reach their peak value, precision rates remain very low which means that even

\begin{tabular}{|c|c|c|c|c|c|c|c|c|c|c|}
\hline \multirow[b]{2}{*}{ Models } & \multicolumn{5}{|c|}{ Building Change Detection } & \multicolumn{4}{|c|}{ Building Semantic Segmentation for 2006} & \multirow{2}{*}{$\begin{array}{l}- \\
\text { Time(sec) }\end{array}$} \\
\hline & Dates & Precision & Recall & F1 & $\overline{\mathbf{B A}}$ & Precision & Recall & F1 & $\overline{\mathbf{B A}}$ & \\
\hline \multirow{3}{*}{$\begin{array}{l}\text { multi-task } \\
\text { FC-Siam-Conc [51] }\end{array}$} & 2 & 44.70 & 59.07 & 50.89 & 79.28 & 74.41 & 65.92 & 69.91 & 82.36 & $\approx 14$ \\
\hline & 3 & 46.96 & 57.74 & 51.79 & 78.65 & 75.15 & 63.14 & 68.63 & 81.02 & $\approx 14$ \\
\hline & 5 & 50.23 & 57.68 & 53.70 & 78.65 & 78.78 & 57.12 & 66.22 & 78.15 & $\approx 15$ \\
\hline \multirow{3}{*}{$\begin{array}{l}\text { multi-task } \\
\text { FC-Siam-Diff [51] }\end{array}$} & 2 & 44.09 & 62.11 & 51.57 & 80.79 & 75.90 & 63.50 & 69.15 & 81.21 & $\approx 14$ \\
\hline & 3 & 45.18 & 59.72 & 51.44 & 79.61 & 75.39 & 64.00 & 69.23 & 81.44 & $\approx 14$ \\
\hline & 5 & 41.87 & 48.92 & 45.12 & 74.23 & 73.79 & 66.81 & 70.13 & 82.78 & $\approx 15$ \\
\hline \multirow{2}{*}{ Method in [59] } & 2 & 47.02 & 56.83 & 51.46 & 78.20 & 76.34 & 63.38 & 69.25 & 81.17 & $\approx 14$ \\
\hline & 5 & 40.67 & 58.96 & 48.14 & 79.19 & 75.11 & 63.77 & 68.98 & 81.32 & $\approx 15$ \\
\hline \multirow{2}{*}{ Method in [61] } & 2 & 51.97 & 49.61 & 50.76 & 74.65 & 74.41 & 66.07 & 70.00 & 82.43 & $\approx 14$ \\
\hline & 5 & 42.46 & 42.82 & 42.64 & 71.21 & 74.41 & 66.07 & 70.00 & 82.43 & $\approx 15$ \\
\hline \multirow{3}{*}{$\begin{array}{l}\text { Ours } \\
\text { multi-task L-UNet }\end{array}$} & 2 & 44.53 & 61.39 & 51.62 & 80.44 & 67.38 & 75.54 & 71.23 & 86.80 & $\approx 15$ \\
\hline & 3 & 46.89 & 61.07 & 53.05 & 80.30 & 75.48 & 61.48 & 67.77 & 80.21 & $\approx 15$ \\
\hline & 5 & 52.42 & 59.68 & 55.82 & 79.65 & 76.08 & 61.52 & 68.03 & 80.24 & $\approx 18$ \\
\hline
\end{tabular}

TABLE (IV) Quantitative evaluation of the proposed multi-task L-UNet on the testing part of Attica VHR dataset. On the left part, the evaluation of the change detection task is presented, while on the right part, metrics for the building semantic segmentation are provided. Precision, recall and F1 rates are associated to the change class as well as the building class for 2006, while Balanced Accuracy (BA) is also provided. All the rows demonstrate results using the RGB-NIR bands with the last column indicating the time needed by each method to produce annotations for a testing image of dimensions $1200 \times 1300$. 


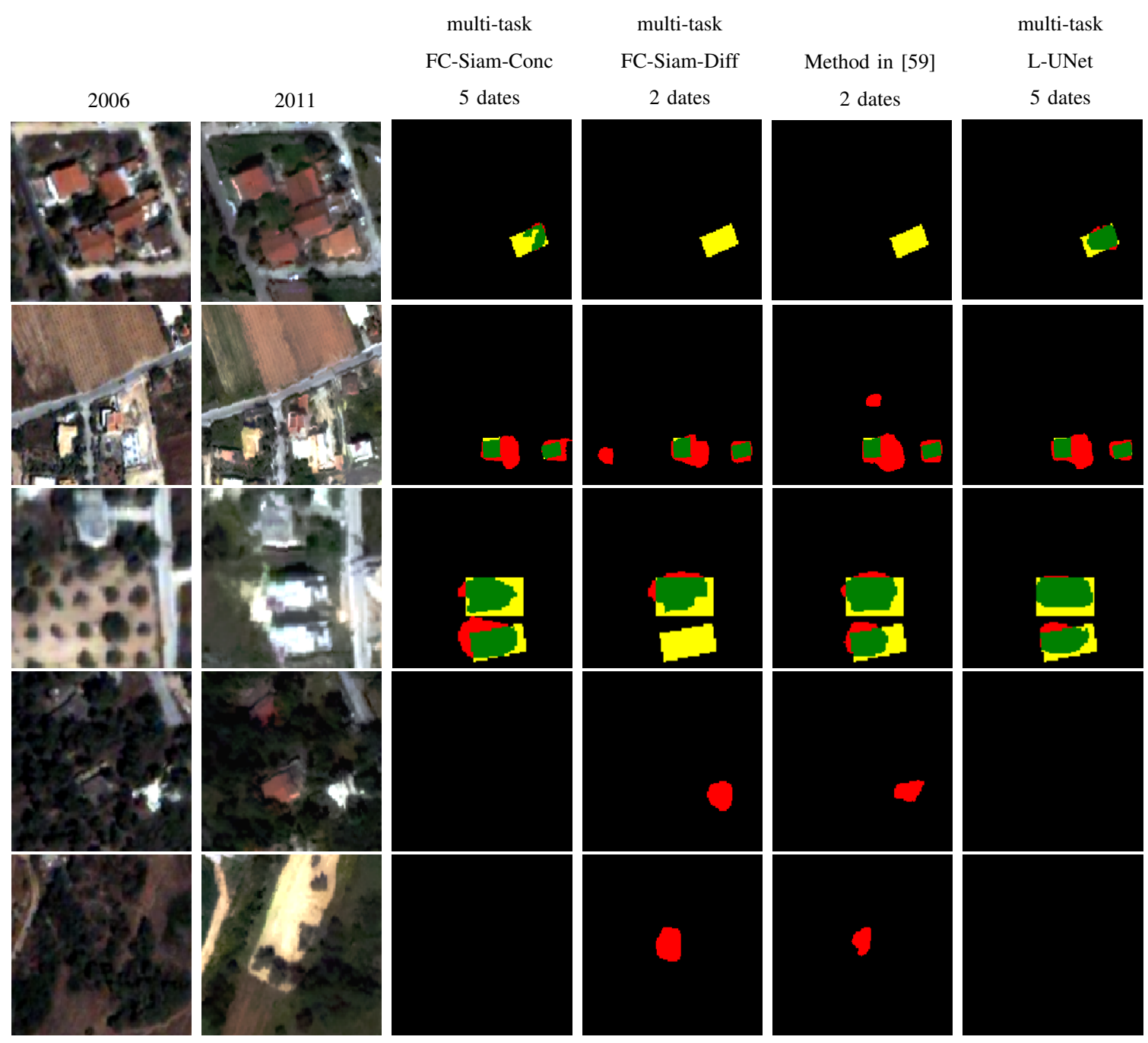

Fig. (9) Qualitative evaluation of the proposed multi-task L-UNet on zoomed regions of the Attica VHR testing areas for the change detection task. 1st column: RGB images of 2006, 2nd column: RGB images of 2011, 3rd column: multi-task FC-Siam-Conc with 5 dates, 4th column: multi-task FC-Siam Diff with 2 dates, 5th column: Method in [59] with 2 dates, 6th column: multi-task L-UNet with 5 dates [Green: True Positives, Black: True Negatives, Red: False Positives, Yellow: False Negatives]

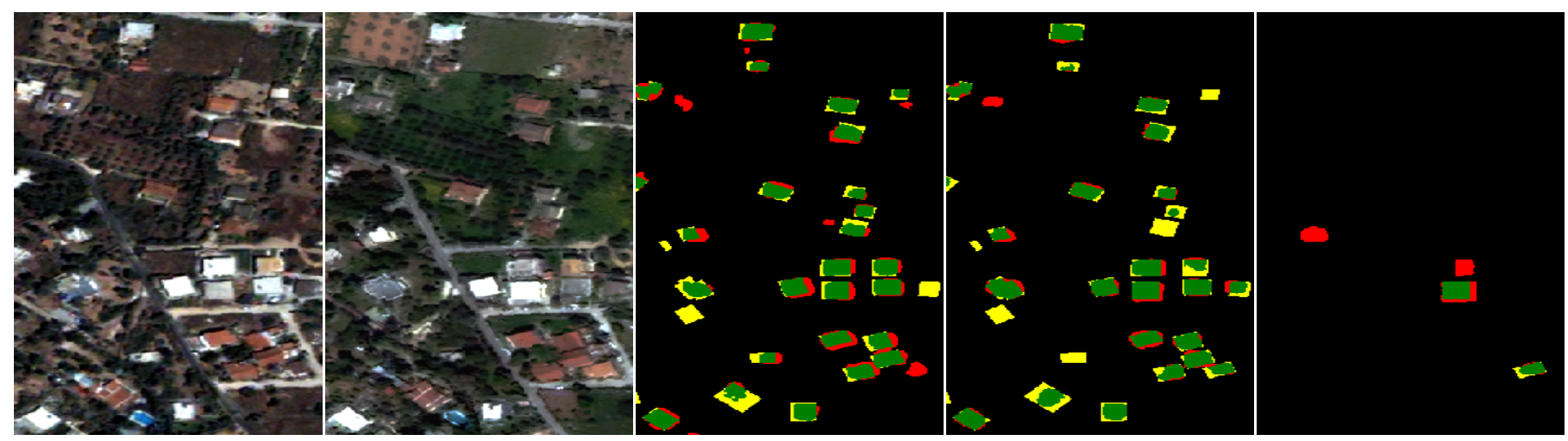

Fig. (10) Qualitative results of multi-task L-UNet with 5 dates, for a region of Attica VHR testing part. From left to right: RGB image of 2006, RGB image of 2011, building predictions of 2006, building predictions of 2011, change predictions. [Green: True Positives, Black: True Negatives, Red: False Positives, Yellow: False Negatives]

though false negative pixels are more limited, many false positive pixels continue to exist.

Continuing with the bi-temporal multi-task learning approaches in [59], [61], it seems that they report higher preci- sion rates compared with the corresponding bi-temporal cases of [51], with [59] achieving a higher F1 score than [61]. As the dates rise however, these methods result in lower performance, as shown in the 5 dates case. The best performance concerning 
false positive rates is attained by the proposed multi-task L-UNet. Specifically, the precision rate reaches $52.42 \%$ in the case of 5 dates, exceeding the second highest precision score of multi-task FC-Siam-Conc by approximately $2.2 \%$. In addition, the F1 score becomes equal to $55.82 \%$ which is also $2.2 \%$ higher than the corresponding F1 rate in the multitask FC-Siam-Conc case. In the proposed approach the F1 score remains always above $50 \%$ which means that temporal attributes in combination with the additional features of the semantic segmentation task, boost greatly the performance of the network attaining a more balanced total number of false positive and false negative pixels. Looking back at Table III, we can further realize the great benefits of the multi-task setting for the L-UNet, since in Table IV precision and F1 rates have rised by $4.5 \%$ and $2.4 \%$ respectively.

Regarding semantic segmentation on buildings, the provided accuracy metrics are related to the year of 2006. The highest precision rate is achieved by multi-task FC-Siam-Conc with 5 dates, while the rest of the accuracy scores are better in the case of multi-task L-UNet with 2 dates. In order to assess the performance of multi-task L-UNet on the semantic segmentation task and in particular on the building footprint detection, we compare it with the performance of a standard U-Net architecture that is commonly used for this problem. The evaluation of the standard U-Net on the testing images of 2006 resulted in $80.15 \%, 60.91 \%, 69.22 \%$ and $80.05 \%$ for precision, recall, F1 and balanced accuracy respectively. Looking at Table IV which summarises the evaluation for the building footprints of 2006 using the multi-task networks,

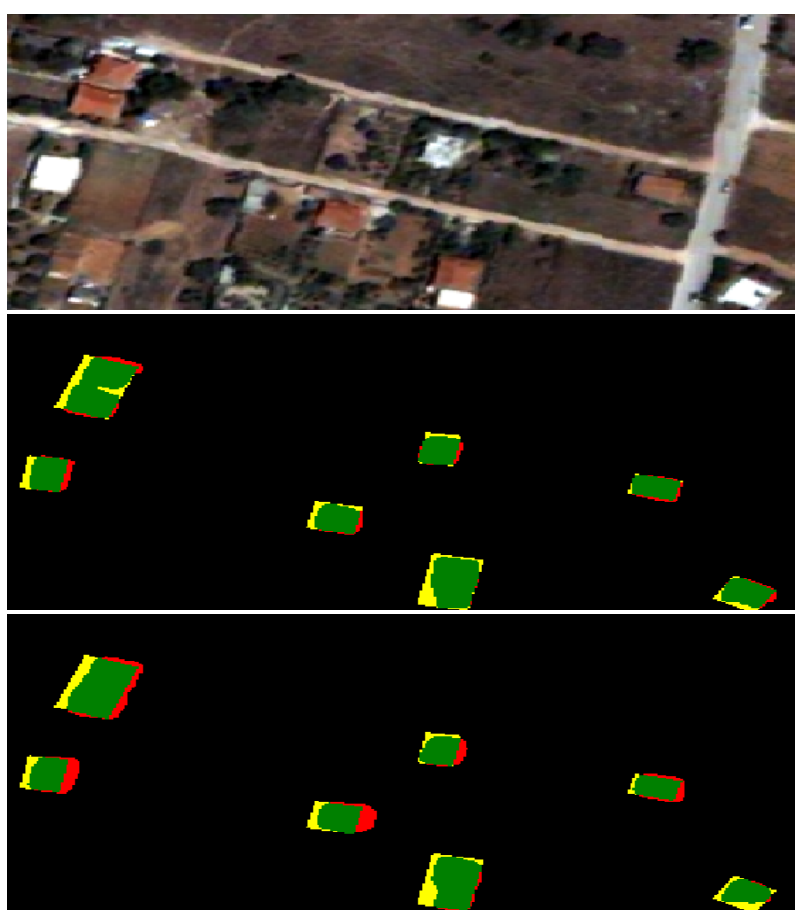

Fig. (11) Qualitative evaluation on building predictions of 2006. From top to bottom: RGB image of 2006, building semantic segmentation with standard U-Net, multi-task L-UNet with 5 dates [Green: True Positives, Black: True Negatives, Red: False Positives, Yellow: False Negatives] one can notice that all evaluation metrics except precision can achieve higher values when combined with the change detection task. This indicates that our formulation boosts the performance of each individual task by fusing together useful features from each problem. From a qualitative perspective, the building predictions resulting from the multi-task framework are quite similar with those resulting from the semantic segmentation task alone. In Figure 11 we can observe building predictions of 2006 using the multi-task L-UNet with 5 dates as well as the standard U-Net.

As a whole, precision values never exceed the rate of $53 \%$ for the change detection task, indicating the more challenging nature of the complex very high resolution images compared to the high resolution ones. Two are the principal reasons that constitute this problem; registration and parallax errors that perplex the learning procedure as well as the different types of change that are included in the satellite images. The variety of changes (e.g land use diversification, alterations in vegetation) results in a wide range of spectral values for certain areas where urban change does not take place. Another fundamental problem which hinders the successful learning process is that change and no change categories are greatly disproportionate. For the Attica VHR dataset, the total number of no change pixels for the training dataset is almost 85 times larger than the number of change ones. Notwithstanding these difficulties, the L-UNet method seems to fully exploit the available information.

Continuing with the qualitative evaluation, in the first row of Figure 9 one can notice that the proposed approach has detected more accurately the additional building compared to the rest of the methods. Continuing with the second row, we can observe that even though all methods are confused by certain rooftop illumination changes, the approaches employing 5 dates have resulted in less false positive values. The third row demonstrates a case where the total number of false positive and false negative pixels is lower for multi-task LUNet with 5 dates. Finally, the last two rows display instances where methods utilizing additional dates deal with rooftop and vegetation alterations in a more constructive way.

One problem that is evident from the qualitative evaluation is that of inconsistent building boundaries. All employed methods regardless of their level of success in identifying the urban changes usually fail to provide accurate boundaries resulting in many false positive pixels along the perimeter of buildings. This issue can also be noticed in Figure 10 where the building predictions of multi-task L-UNet for both 2006 and 2011 are provided along with the corresponding change for a larger testing region of the Attica VHR dataset.

As far as the SpaceNet7 dataset is concerned, numerical results of the conducted experiments are outlined in Table V. Starting with the methods presented in [51], FC-Siam-Conc has achieved the highest F1 score when employing 2 dates, while FC-EF attained the best balanced accuracy in the case of 10 dates. The corresponding multi-task results seem to be better only for the FC-Siam-Conc case since F1 score has raised from $44.80 \%$ to $45.16 \%$. On the contrary, accuracy metrics for FC-Siam-Diff did not benefit much neither from the additional dates, nor from the multi-task setting. In the FC- 


\begin{tabular}{lllllll}
\hline Models & Dates & Precision & Recall & F1 & BA & Time(sec) \\
\hline \multirow{2}{*}{ FC-Siam-Conc [51] } & 2 & 39.07 & 52.50 & 44.80 & 75.84 & $\approx 8$ \\
& 10 & 42.22 & 38.97 & 40.53 & 69.22 & $\approx 10$ \\
\hline multi-task & 2 & 34.41 & 55.78 & 42.56 & 77.36 & $\approx 8$ \\
FC-Siam-Conc [51] & 10 & 38.70 & 54.20 & 45.16 & 76.67 & $\approx 10$ \\
\hline \multirow{2}{*}{ FC-Siam-Diff [51] } & 2 & 29.74 & 48.00 & 36.73 & 73.43 & $\approx 8$ \\
& 10 & 31.84 & 53.11 & 39.81 & 75.99 & $\approx 10$ \\
\hline multi-task & 2 & 29.78 & 46.79 & 36.40 & 72.84 & $\approx 8$ \\
FC-Siam-Diff [51] & 10 & 22.66 & 24.59 & 23.59 & 61.88 & $\approx 10$ \\
\hline \multirow{2}{*}{ FC-EF [51] } & 2 & 42.96 & 42.68 & 42.82 & 71.06 & $\approx 8$ \\
& 10 & 34.29 & 57.71 & 43.02 & 78.30 & $\approx 10$ \\
\multirow{2}{*}{ Method in [59] } & 2 & 21.19 & 60.72 & 31.41 & 79.23 & $\approx 8$ \\
& 10 & 26.15 & 60.99 & 36.61 & 79.63 & $\approx 10$ \\
\multirow{2}{*}{ Method in [61] } & 2 & 24.19 & 61.84 & 34.77 & 79.95 & $\approx 8$ \\
\multirow{2}{*}{ LSTM [55] } & 10 & 31.97 & 55.85 & 40.66 & 77.33 & $\approx 10$ \\
Method in [69] & 10 & 38.75 & 48.76 & 43.18 & 74.00 & $\approx 13$ \\
\multirow{2}{*}{ L-UNet } & 10 & 34.87 & 54.72 & 42.59 & 76.85 & $\approx 13$ \\
\hline \multirow{2}{*}{ multi-task L-UNet } & 2 & 36.42 & 56.46 & 44.28 & 77.74 & $\approx 9$ \\
& 10 & 44.83 & 53.82 & 48.92 & 76.58 & $\approx 12$ \\
\hline
\end{tabular}

TABLE (V) Quantitative evaluation of L-UNet and multi-task LUNet on the testing part of SpaceNet7 dataset. Precision, recall and F1 rates are associated to the change class, while Balanced Accuracy (BA) is also provided. All the rows demonstrate results using the RGB-NIR bands with the last column indicating the time needed by each method to produce annotations for a testing image of dimensions $1024 \times 1024$.

EF case, all accuracy metrics except precision are ameliorated when more dates are used. Continuing with the multi-task approaches proposed in [59] and [61], one can notice that they have produced many false positive values since the precision rates are very low. The highest F1 score has resulted from the method in [61] when utilizing 10 dates. Regarding the LSTM [55] and the method in [69] which have been proposed for time-series datasets, we can observe that accuracy rates are quite similar between the two approaches. Compared with the rest of the approaches, the time-series methods provide better results than the multi-task ones [59], [61], but similar results with the methods in [51]. Finally, L-UNet boosts the precision and $\mathrm{F} 1$ rates when we take advantage of more dates. In the mulit-task L-UNet framework, recall rate and balanced accuracy reach the highest level in the case of 2 dates, while precision and F1 scores become optimal in the case of 10 dates. As a whole, in this dataset the proposed multi-task LUNet outperforms all other methods for all accuracy metrics. Especially, when more temporal information is integrated, precision and F1 scores benefit the most by the suggested method, exceeding the rest of the approaches by at least $4.9 \%$. As far as the results on building semantic segmentation are concerned, we evaluated the multi-task models on the first date of SpaceNet7. The highest F1 score and balanced accuracy resulted from the [59] and were equal to $47.14 \%$ and $77.30 \%$ respectively. The F1 score attained by the multi-task L-UNet was similar and equal to $46.79 \%$ while the balanced accuracy was $73.24 \%$.

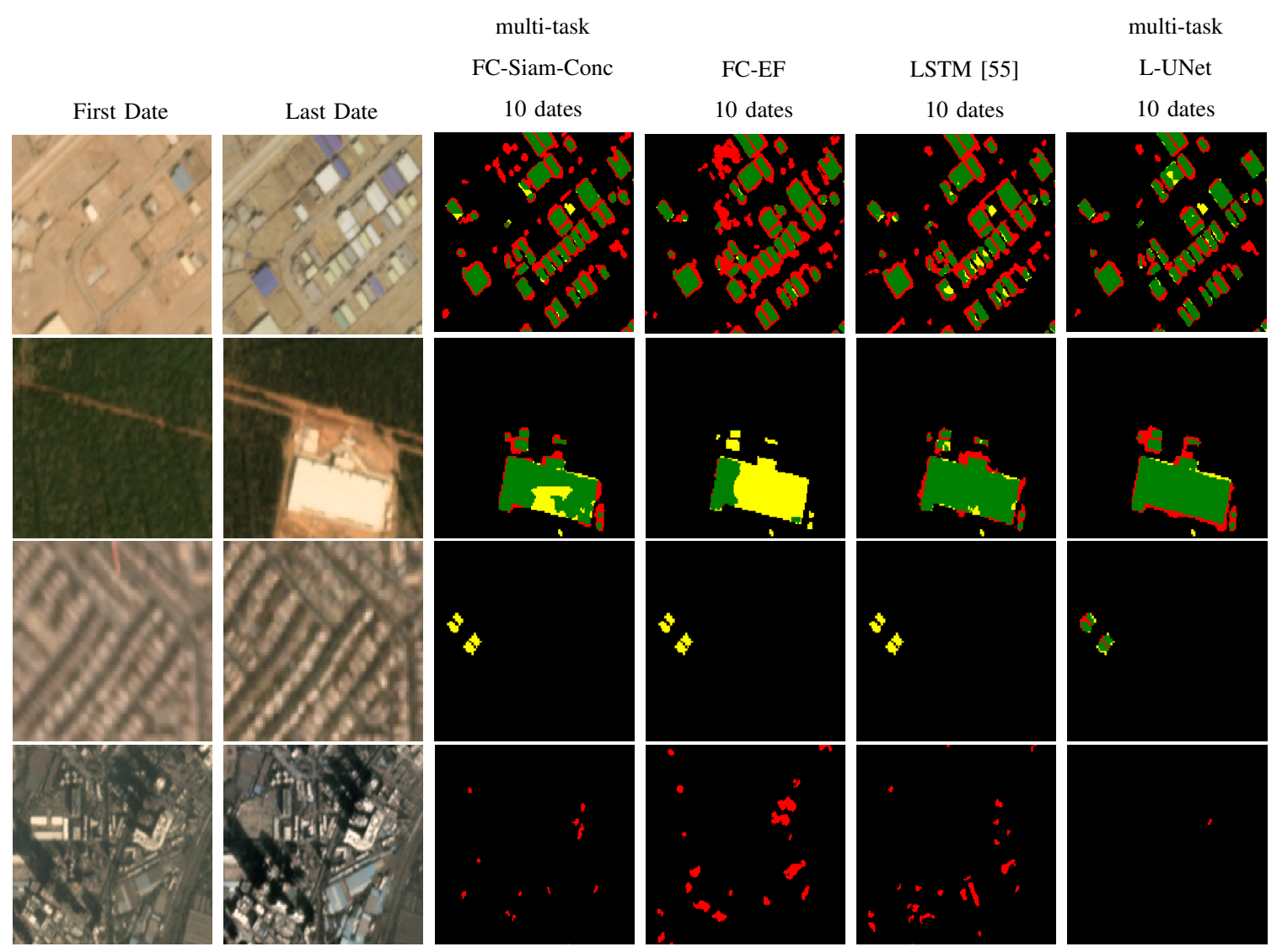

Fig. (12) Qualitative evaluation of the proposed multi-task L-UNet on zoomed regions of the SpaceNet7 testing areas for the change detection task. 1st column: RGB images of the first date, 2nd column: RGB images of the last date, 3rd column: multi-task FC-Siam-Conc with 10 dates, 4th column: FC-EF with 10 dates, 5th column: LSTM [55] with 10 dates, 6th column: multi-task L-UNet with 10 dates [Green: True Positives, Black: True Negatives, Red: False Positives, Yellow: False Negatives] 
Qualitative samples from the testing part of SpaceNet7 dataset are delineated in Figure 12 for some of the investigated approaches. In the first row, we can see that even though all methods produce false positive pixels between the building boundaries, multi-task L-UNet has resulted in a more clear separation of the buildings. In the second row, the LSTM network [55] as well as the proposed multi-task L-UNet have identified better the main building footprint of the image. Regarding the third row, here the multi-task L-UNet has managed to detect the construction of some small buildings. Finally, the last row shows an example where the proposed approach includes the less false positive pixels caused by building shadows and illumination differences.

Regarding inference time, similarly to L-UNet, we can notice that multi-task L-UNet does not need much more time to produce annotations, compared with the rest of the methods.

\section{Evaluation of the different segmentation and change de- tection loss components}

In this subsection, we conduct an ablation study and we discuss the importance of each of the loss components for the coupling of semantic segmentation and change detection tasks. The performance of these components is reported on the Attica VHR and SpaceNet7 datasets, on which annotations for both the building class and the urban change class are available. For this evaluation, we chose the best performing model (multitask L-UNet with 5 dates for the Attica VHR dataset, and multi-task L-UNet with 10 dates for the SpaceNet7 dataset) and trained it using different loss compositions, as described in the caption of Figure 13. Giving some more details, Ablation1 represents multi-task L-UNet with 5 dates using 3 losses; one for the change detection task and two for the building semantic segmentation of the first and last available dates. In Ablation2 the additional $\operatorname{Loss}_{c h 2}$ is employed, while in Abla-

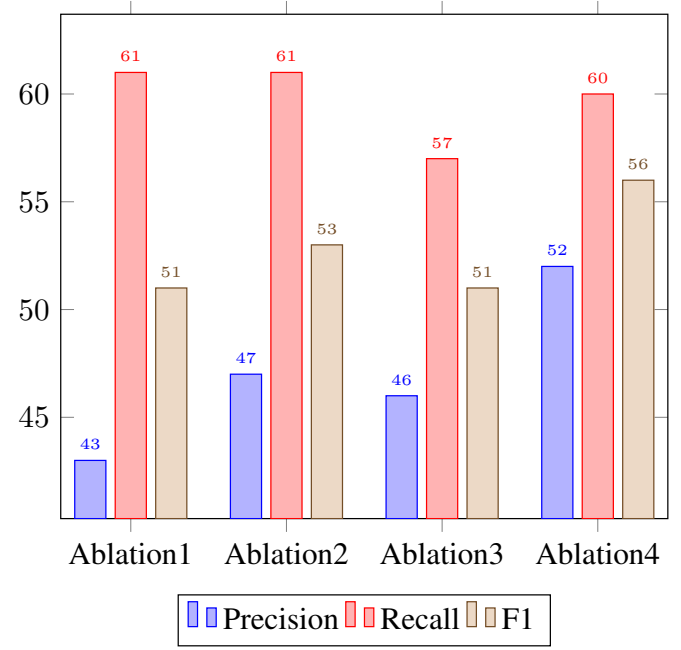

Fig. (13) Accuracy metrics for multi-task L-UNet with 5 dates using different combinations of losses. The provided metrics have resulted from the testing part of Attica VHR dataset. Ablation1 $\left[\operatorname{Loss}_{\text {ch }}, \operatorname{Loss}_{\text {seg }}^{1}, \operatorname{Loss}_{\text {seg }}^{T}\right]$, Ablation2 [Loss ${ }_{\text {ch }}, \operatorname{Loss}_{\text {seg }}^{1}, \operatorname{Loss}_{\text {seg }}^{T}$, $\left.\operatorname{Loss}_{c h 2}\right]$, Ablation3 [Loss $\left.{ }_{c h}, \operatorname{Loss}_{\text {seg }}^{1}, \operatorname{Loss}_{\text {seg }}^{T}, \operatorname{Loss}_{\text {seg2 }}^{T}\right]$, Ablation4 $\left[\operatorname{Loss}_{\text {ch }}\right.$, Loss $_{\text {seg }}^{1}$, Loss seg $_{\text {seg }}^{T}$, Loss $_{\text {ch } 2}$, Loss $\left._{\text {seg } 2}^{T}\right]$.

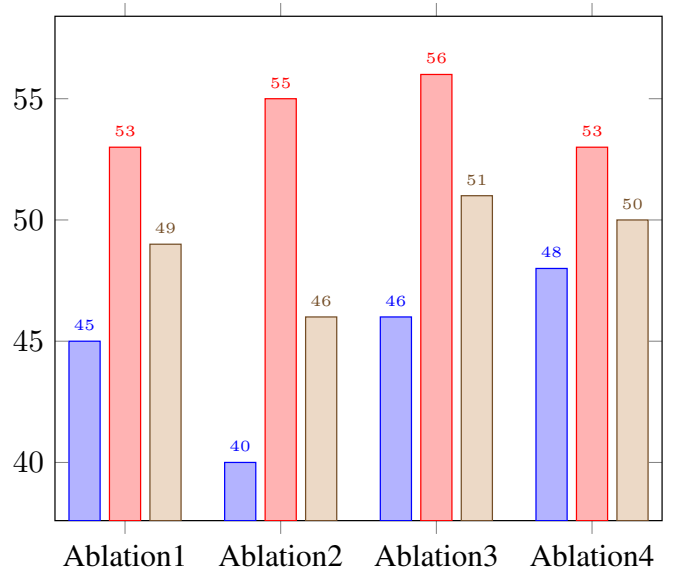

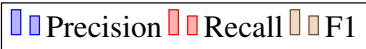

Fig. (14) Accuracy metrics for multi-task L-UNet with 10 dates using different combinations of losses. The provided metrics have resulted from the testing part of SpaceNet7 dataset. Ablation1 $\left[\operatorname{Loss}_{\text {ch }}, \operatorname{Loss}_{\text {seg }}^{1}, \operatorname{Loss}_{\text {seg }}^{T}\right]$, Ablation2 $\left[\operatorname{Loss}_{\text {ch }}, \operatorname{Loss}_{\text {seg }}^{1}, \operatorname{Loss}_{\text {seg }}^{T}\right.$, $\operatorname{Loss}_{\text {ch } 2}$ ], Ablation3 [Loss $\left.{ }_{c h}, \operatorname{Loss}_{\text {seg }}^{1}, \operatorname{Loss}_{\text {seg }}^{T}, \operatorname{Loss}_{\text {seg } 2}^{T}\right]$, Ablation4 $\left[\operatorname{Loss}_{\text {ch }}, \operatorname{Loss}_{\text {seg }}^{1}, \operatorname{Loss}_{\text {seg }}^{T}, \operatorname{Loss}_{\text {ch } 2}, \operatorname{Loss}_{\text {seg2 }}^{T}\right]$.

tion3 $\operatorname{Loss}_{\text {seg } 2}^{T}$ is used as the additional fourth loss component. Finally, Ablation4 includes all five losses as described in section II-C, resulting to our proposed framework. After training each of the different frameworks, the evaluation metrics were calculated on the test part of Attica VHR and SpaceNet7 datasets. Looking at Figure 13 for the Attica VHR dataset, we can observe that recall rates are almost the same in Ablation 1, 2 and 4, while in Ablation3 a lower value is attained, meaning that the integration of $\operatorname{Loss}_{\operatorname{seg} 2}^{T}$ alone produces a deteriorated result regarding false negative detections. As far as precision rates are concerned, they increase from $43 \%$ to $47 \%$ when

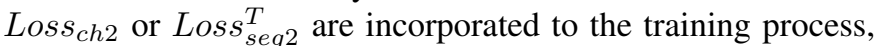
indicating the contribution of the additional circular losses to the lessening of false positive values. The amelioration of false positive pixels is even more obvious when both circular losses are integrated, with precision rate reaching the value of 52\%. In this case, F1 score also reaches the highest level, becoming equal to $56 \%$.

Regarding the SpaceNet7 dataset, the ablation study results are shown in Figure 14. In this setting, we can observe that Ablation1 has a lower precision rate than Ablation4, meaning that $L_{o s s} s_{c h 2}$ and $\operatorname{Loss}_{s e g 2}^{T}$ contribute to the lessening of false positive detections. In Ablation2, the recall rate has rized but precision has become very low, leading also to a lower F1 score. Finally, in Ablation3 the results are similar with Ablation 4 but with a wider difference between the precision and recall rates. Once again in this case, we notice that Ablation 4 results in the lowest number of false positive detections, benefiting from the combination of all the loss components.

\section{E. Discussion}

Taking into consideration all the conducted experiments on all the different datasets, we can draw some conclusions about the investigated methods. Overall, the integration of LSTM 
networks as skip connections provides efficient aggregation strategies able to encode information presented on two or more temporal time stamps. Results indicated that such an approach can outperform other aggregation functions like concatenation and early fusion. Subtraction was the least efficient when more temporal information was incorporated. In the case of concatenation and subtraction, the multi-task setting can boost the performances, however without exceeding the results of the proposed formulation. In fact, our method attains the highest precision and F1 rates in all dataset cases, while in the SpaceNet7 case, all accuracy metrics reach the highest scores. Concerning the compared bi-temporal multi-task methods, they seem to have difficulty in processing successfully the additional temporal information, whereas the suggested method benefits greatly not only from the supplementary temporal features as well as from the multi-task setting, outperforming at the same time the rest of the time-series methods. Our method reports stable and higher performance on the precision rate and F1 score, contributing a lot to the lessening of false positive detections.

Change detection applications suffer greatly from the numerous false positive detections that arise from registration errors, illumination differences, or other types of change unrelated to the problem of interest. Hence, the development of proper formulas that overcome this obstacle becomes necessary. In the L-UNet case, the multi-task setting contributes greatly to the amelioration of the results compared with the single-task change detection framework. Specifically, precision and F1 scores are greatly improved, benefiting both from the supplementary segmentation features and the circular losses. The superiority of the method has been proven in three completely different datasets with different spatial and temporal resolutions. In the future, we would like to investigate also the impact of more than two semantic categories to the proposed formulation both in terms of model complexity and evaluation performance.

One interesting direction that could be further investigated in the multi-temporal setting is the integration of the time that the change has occurred in the time series. Determining the time point that an urban change firstly emerges can be very useful for tracking purposes, providing more thorough information for the frequency of urbanization. For our experiments, the change annotation of all the employed datasets describes the changes that have occurred between the first and the last date without exploiting additional information about the exact time of the change. Such a setup may lead to concerns as to whether a deep learning based architecture can handle in a constructive way this irregular distribution of the changes through the different timestamps. Our extensive experiments indicated that the proposed approach boosts the performance when more dates are employed. This encourages us to draw the conclusion that the integration of LSTMs in the different encoding parts can provide meaningful temporal feature vectors independent of the specific time of change.

Given a time-series dataset, if we want to determine the exact point of change using LSTMs, one solution can be to pass the multi-temporal images to a convolutional LSTM encoder as in [55], and produce detection scores for every LSTM cell output $h_{t}$ [70]. That is, passing $h_{t}$ to a convolutional layer for classification and then apply a softmax function to extract the semantic probability map. If we can properly optimize such an approach, we will end up with distinct semantic maps for every single date, meaning that we can compose a tracking map and determine when each object appears for the first time. Another interesting approach is to concentrate on the internal activations of the LSTM cell, namely the operations of the internal gates as well as the information stored in the cell state. According to [71], the cell state is able to keep important information across consecutive time steps, concerning the changed parts of the current sequential image pair. Hence, with proper fine-tuning it may be able to recognize the changes after every time step. For example, in [55] the activations of the cell state as well as the internal gates have helped the model to identify and discard the cloud regions, operating as filter mechanisms.

\section{CONCLUSION}

In this paper, a novel multi-task learning framework for urban change detection is proposed where fully convolutional LSTM blocks are integrated on top of every encoding level of a U-Net like deep architecture, while an additional decoding branch is utilized for the semantic segmentation of the first and last employed dates. By using such a framework, temporal relationships are calculated for feature vectors of various resolutions without the need to downsample or flatten them. At the same time, the extra decoder provides supplementary information concerning semantic categories. Quantitative and qualitative analyses indicated that even though the problem of change detection can be very challenging due to illumination differences and registration errors, more constrained formulations and multi-task deep learning frameworks based on LSTMs can provide very good tools for it. In fact, the proposed method contributed greatly to the lessening of false positive values boosting significantly the precision and F1 rates in all dataset cases. In the future, we plan to further evolve the proposed formula by performing simultaneously image registration and change detection, in order to eliminate parallax errors that tend to disorientate the learning process during training. In addition, we will explore ways to determine the change timestamps based on the operations of the LSTM networks. Finally, we will try to ameliorate and preserve the shape of the detected objects.

\section{REFERENCES}

[1] A. SINGH, "Review article digital change detection techniques using remotely-sensed data," International Journal of Remote Sensing, vol. 10, no. 6, pp. 989-1003, 1989. [Online]. Available: https: //doi.org/10.1080/01431168908903939

[2] K. Karantzalos, "Recent advances on $2 \mathrm{~d}$ and $3 \mathrm{~d}$ change detection in urban environments from remote sensing data," Computational Approaches for Urban Environments, pp. 237-272, 112015.

[3] R. J. Radke, S. Andra, O. Al-Kofahi, and B. Roysam, "Image change detection algorithms: a systematic survey," IEEE Transactions on Image Processing, vol. 14, pp. 294-307, 2005.

[4] L. Gómez-Chova, D. Tuia, G. Moser, and G. Camps-Valls, "Multimodal classification of remote sensing images: A review and future directions," Proceedings of the IEEE, vol. 103, pp. 1560-1584, 2015. 
[5] N. Longbotham, F. Pacifici, T. C. Glenn, A. Zare, M. Volpi, D. Tuia, E. Christophe, J. Michel, J. Inglada, J. Chanussot, and Q. Du, "Multimodal change detection, application to the detection of flooded areas: Outcome of the 2009-2010 data fusion contest," IEEE Journal of Selected Topics in Applied Earth Observations and Remote Sensing, vol. 5, pp. 331-342, 2012.

[6] J. Yang, P. Weisberg, and N. A Bristow, "Landsat remote sensing approaches for monitoring long-term tree cover dynamics in semiarid woodlands: Comparison of vegetation indices and spectral mixture analysis," Remote Sensing of Environment, vol. 119, 022012.

[7] B. Liang and Q. Weng, "Assessing urban environmental quality change of indianapolis, united states, by the remote sensing and gis integration," Selected Topics in Applied Earth Observations and Remote Sensing, IEEE Journal of, vol. 4, pp. 43 - 55, 042011.

[8] A. Taneja, L. Ballan, and M. Pollefeys, "City-scale change detection in cadastral 3d models using images," 2013 IEEE Conference on Computer Vision and Pattern Recognition, pp. 113-120, 2013.

[9] P. Singh, Z. Kato, and J. Zerubia, "A multilayer Markovian model for change detection in aerial image pairs with large time differences," in IARP International Conference on Pattern Recognition, IAPR. Stockholm, Sweden: IEEE, Aug. 2014, accepted.

[10] C. Benedek, M. Shadaydeh, Z. Kato, T. Szirányi, and J. Zerubia, "Multilayer Markov Random Field Models for Change Detection in Optical Remote Sensing Images," ISPRS Journal of Photogrammetry and Remote Sensing, vol. 107, pp. 22-37, 2015. [Online]. Available: https://hal.inria.fr/hal-01116609

[11] M. Volpi, D. Tuia, G. Camps-Valls, and M. F. Kanevski, "Unsupervised change detection with kernels," IEEE Geoscience and Remote Sensing Letters, vol. 9, pp. 1026-1030, 2012.

[12] M. Vakalopoulou, K. Karantzalos, N. Komodakis, and N. Paragios, "Simultaneous registration and change detection in multitemporal, very high resolution remote sensing data," 2015 IEEE Conference on Computer Vision and Pattern Recognition Workshops (CVPRW), pp. 61-69, 2015.

[13] M. Vakalopoulou, C. Platias, M. Papadomanolaki, N. Paragios, and K. Karantzalos, "Simultaneous registration, segmentation and change detection from multisensor, multitemporal satellite image pairs," 2016 IEEE International Geoscience and Remote Sensing Symposium (IGARSS), pp. 1827-1830, 2016.

[14] J. Deng, K. Wang, Y. H. Deng, and G. J. Qi, "Pca-based land-use change detection and analysis using multitemporal and multisensor satellite data," International Journal of Remote Sensing - INT J REMOTE SENS, vol. 29 , pp. 4823-4838, 082008.

[15] X. Li and A. G.-O. Yeh, "Principal component analysis of stacked multitemporal images for the monitoring of rapid urban expansion in the pearl river delta," International Journal of Remote Sensing, vol. 19, no. 8, pp. $1501-1518,1998$

[16] X. Huang, C. Yinxia, and J. Li, "An automatic change detection method for monitoring newly constructed building areas using time-series multiview high-resolution optical satellite images," Remote Sensing of Environment, vol. 244, p. 111802, 072020.

[17] L. Xuecao, Y. Zhou, Z. Zhu, L. Liang, B. Yu, and W. Cao, "Mapping annual urban dynamics (1985-2015) using time series of landsat data," Remote Sensing of Environment, vol. 216, 082018.

[18] X.-P. Song, J. Sexton, C. Huang, S. Channan, and J. Townshend, "Characterizing the magnitude, timing and duration of urban growth from time series of landsat-based estimates of impervious cover," Remote Sensing of Environment, vol. 175, pp. 1-13, 2016.

[19] F. Pacifici and F. Del Frate, "Automatic change detection in very high resolution images with pulse-coupled neural networks," Geoscience and Remote Sensing Letters, IEEE, vol. 7, pp. 58 - 62, 022010.

[20] C. Pratola, F. Del Frate, G. Schiavon, and D. Solimini, "Toward fully automatic detection of changes in suburban areas from vhr sar images by combining multiple neural-network models," IEEE Transactions on Geoscience and Remote Sensing, vol. 51, pp. 2055-2066, 022013.

[21] S. Stent, R. Gherardi, B. Stenger, and R. Cipolla, "Detecting change for multi-view, long-term surface inspection," in BMVC, 2015.

[22] J. Liu, M. Gong, K. Qin, and P. Zhang, "A deep convolutional coupling network for change detection based on heterogeneous optical and radar images," IEEE Transactions on Neural Networks and Learning Systems, vol. PP, pp. 1-15, 122016.

[23] M. Gong, J. Zhao, J. Liu, Q. Miao, and L. Jiao, "Change detection in synthetic aperture radar images based on deep neural networks," IEEE transactions on neural networks and learning systems, vol. 27, 062015.

[24] A. M. El Amin, Q. Liu, and Y. Wang, "Zoom out cnns features for optical remote sensing change detection," in 2017 2nd International Conference on Image, Vision and Computing (ICIVC), June 2017, pp. 812-817.
[25] Y. Zhan, K. Fu, M. Yan, X. Sun, H. Wang, and X. Qiu, "Change detection based on deep siamese convolutional network for optical aerial images," IEEE Geoscience and Remote Sensing Letters, vol. PP, pp. 1-5, 082017.

[26] A. Krizhevsky, I. Sutskever, and G. E. Hinton, "Imagenet classification with deep convolutional neural networks," Neural Information Processing Systems, vol. 25, 012012.

[27] M. Vakalopoulou, G. Chassagnon, N. Bus, R. Marini, E. I. Zacharaki, M.-P. Revel, and N. Paragios, "Atlasnet: Multi-atlas non-linear deep networks for medical image segmentation," in MICCAI, 2018.

[28] H. Schwenk, L. Barrault, A. Conneau, and Y. LeCun, "Very deep convolutional networks for text classification," in EACL, 2016.

[29] J. Y.-H. Ng, J. Choi, J. Neumann, and L. S. Davis, "Actionflownet: Learning motion representation for action recognition," 2018 IEEE Winter Conference on Applications of Computer Vision (WACV), pp. 1616-1624, 2016.

[30] S. Saha, Y. T. Solano-Correa, F. Bovolo, and L. Bruzzone, "Unsupervised deep transfer learning-based change detection for hr multispectral images," IEEE Geoscience and Remote Sensing Letters.

[31] S. Saha, L. Mou, X. X. Zhu, F. Bovolo, and L. Bruzzone, "Semisupervised change detection using graph convolutional network," IEEE Geoscience and Remote Sensing Letters, 2020.

[32] M. Vakalopoulou, K. Karantzalos, N. Komodakis, and N. Paragios, "Building detection in very high resolution multispectral data with deep learning features," in 2015 IEEE International Geoscience and Remote Sensing Symposium (IGARSS), 2015.

[33] M. Papadomanolaki, M. Vakalopoulou, and K. Karantzalos, "A Novel Object-Based Deep Learning Framework for Semantic Segmentation of Very High-Resolution Remote Sensing Data: Comparison with Convolutional and Fully Convolutional Networks," Remote Sensing, vol. 11, no. 6, p. 684, Mar. 2019.

[34] M. Papadomanolaki, M. Vakalopoulou, S. Zagoruyko, and K. Karantzalos, "Benchmarking deep learning frameworks for the classification of very high resolution satellite multispectral data," ISPRS Annals of Photogrammetry, Remote Sensing and Spatial Information Sciences, vol. III-7, pp. 83-88, 062016.

[35] B. Huang, K. Lu, N. Audebert, A. Khalel, Y. Tarabalka, J. M. Malof, A. Boulch, B. L. Saux, L. M. Collins, K. Bradbury, S. Lefèvre, and M. El-Saban, "Large-scale semantic classification: Outcome of the first year of inria aerial image labeling benchmark," 2018 IEEE International Geoscience and Remote Sensing Symposium, pp. 6947-6950, 2018.

[36] N. Audebert, B. Le Saux, and S. Lefèvre, "Beyond rgb: Very high resolution urban remote sensing with multimodal deep networks," ISPRS Journal of Photogrammetry and Remote Sensing, 112017.

[37] M. Papadomanolaki, K. Karantzalos, and M. Vakalopoulou, "A multitask deep learning framework coupling semantic segmentation and image reconstruction for very high resolution imagery," in IGARSS, Yokohama, Japan, Jul. 2019.

[38] J. Li, X. Huang, and J. Gong, "Deep neural network for remotesensing image interpretation: status and perspectives," National Science Review, vol. 6, no. 6, pp. 1082-1086, 05 2019. [Online]. Available: https://doi.org/10.1093/nsr/nwz058

[39] J. J. Hopfield, "Neural networks and physical systems with emergent collective computational abilities." Proceedings of the National Academy of Sciences of the United States of America, vol. 79 8, pp. 2554-8, 1982.

[40] D. E. Rumelhart, G. E. Hinton, and R. J. Williams, "Learning representations by back-propagating errors," Nature, vol. 323, pp. 533-536, 1986.

[41] A. Milan, S. H. Rezatofighi, A. R. Dick, I. D. Reid, and K. Schindler, "Online multi-target tracking using recurrent neural networks," in $A A A I$, 2016.

[42] B. Singh, T. K. Marks, M. J. Jones, O. Tuzel, and M. Shao, "A multistream bi-directional recurrent neural network for fine-grained action detection," 2016 IEEE Conference on Computer Vision and Pattern Recognition (CVPR), pp. 1961-1970, 2016.

[43] S. Hochreiter and J. Schmidhuber, "Long short-term memory," Neural Computation, vol. 9, pp. 1735-1780, 1997.

[44] W. Byeon, T. M. Breuel, F. Raue, and M. Liwicki, "Scene labeling with lstm recurrent neural networks," in Proceedings of the IEEE Conference on Computer Vision and Pattern Recognition, 2015, pp. 3547-3555.

[45] K. Ehsani, H. Bagherinezhad, J. Redmon, R. Mottaghi, and A. Farhadi, "Who let the dogs out? modeling dog behavior from visual data," 2018 IEEE/CVF Conference on Computer Vision and Pattern Recognition, pp. 4051-4060, 2018

[46] S. Hochreiter, "The vanishing gradient problem during learning recurrent neural nets and problem solutions," International Journal of Uncertainty, Fuzziness and Knowledge-Based Systems, vol. 6, pp. 107-116, 041998. 
[47] J. Donahue, L. A. Hendricks, M. Rohrbach, S. Venugopalan, S. Guadarrama, K. Saenko, and T. Darrell, "Long-term recurrent convolutional networks for visual recognition and description," IEEE Transactions on Pattern Analysis and Machine Intelligence, vol. 39, pp. 677-691, 2014.

[48] X. Shi, Z. Chen, H. Wang, D.-Y. Yeung, W.-K. Wong, and W. chun Woo, "Convolutional 1stm network: A machine learning approach for precipitation nowcasting," in NIPS, 2015.

[49] J. Chen, L. Yang, Y. Zhang, M. S. Alber, and D. Z. Chen, "Combining fully convolutional and recurrent neural networks for $3 \mathrm{~d}$ biomedical image segmentation," in NIPS, 2016.

[50] R. Caye Daudt, B. Le Saux, A. Boulch, and Y. Gousseau, "Urban change detection for multispectral earth observation using convolutional neural networks," in IEEE International Geoscience and Remote Sensing Symposium (IGARSS), July 2018.

[51] R. Caye Daudt, B. Le Saux, and A. Boulch, "Fully convolutional siamese networks for change detection," in IEEE International Conference on Image Processing (ICIP), October 2018.

[52] L. Mou, L. Bruzzone, and X. xiang Zhu, "Learning spectral-spatialtemporal features via a recurrent convolutional neural network for change detection in multispectral imagery," IEEE Transactions on Geoscience and Remote Sensing, vol. 57, pp. 924-935, 2019.

[53] V. S. F. Garnot, L. Landrieu, S. Giordano, and N. Chehata, "Satellite image time series classification with pixel-set encoders and temporal self-attention," ArXiv, vol. abs/1911.07757, 2019.

[54] M. Rußwurm and M. Körner, "Self-attention for raw optical satellite time series classification," arXiv preprint arXiv:1910.10536, 2019.

[55] M. Rußwurm and M. Körner, "Multi-temporal land cover classification with sequential recurrent encoders," ISPRS International Journal of GeoInformation, vol. 7, 022018.

[56] M. Rußwurm and M. Körner, "Convolutional lstms for cloud-robust segmentation of remote sensing imagery," arXiv preprint arXiv:1811.02471, 2018.

[57] C. Tan, L. Zhao, Z. Yan, K. Li, D. N. Metaxas, and Y. Zhan, "Deep multi-task and task-specific feature learning network for robust shape preserved organ segmentation," 2018 IEEE 15th International Symposium on Biomedical Imaging (ISBI 2018), pp. 1221-1224, 2018.

[58] D. Zhang, J. Han, L. Yang, and D. Xu, "Spftn: A joint learning framework for localizing and segmenting objects in weakly labeled videos," IEEE Transactions on Pattern Analysis and Machine Intelligence, vol. 42, pp. 475-489, 2020.

[59] Y. Liu, C. Pang, Z. Zhan, X. Zhang, and X. Yang, "Building change detection for remote sensing images using a dual task constrained deep siamese convolutional network model," ArXiv, vol. abs/1909.07726, 2019.

[60] T.-Y. Lin, P. Goyal, R. B. Girshick, K. He, and P. Dollár, "Focal loss for dense object detection," 2017 IEEE International Conference on Computer Vision (ICCV), pp. 2999-3007, 2017.

[61] R. C. Daudt, B. L. Saux, A. Boulch, and Y. Gousseau, "Multitask learning for large-scale semantic change detection," Computer Vision and Image Understanding, vol. 187, 2018.

[62] M. Yang, L. Jiao, F. Liu, B. Hou, and S. Yang, "Transferred deep learning-based change detection in remote sensing images," IEEE Transactions on Geoscience and Remote Sensing, vol. 57, pp. 6960-6973, 2019.

[63] A. Radoi and M. Datcu, "Multilabel annotation of multispectral remote sensing images using error-correcting output codes and most ambiguous examples," IEEE Journal of Selected Topics in Applied Earth Observations and Remote Sensing, vol. 12, no. 7, pp. 2121-2134, 2019.

[64] D. Marmanis, J. Wegner, S. Galliani, K. Schindler, M. Datcu, and U. Stilla, "Semantic segmentation of aerial images with an ensemble of cnns," ISPRS Annals of Photogrammetry, Remote Sensing and Spatial Information Sciences, vol. III-3, pp. 473-480, 062016.

[65] L. Mou, Y. Hua, and X. X. Zhu, "Relation matters: Relational contextaware fully convolutional network for semantic segmentation of highresolution aerial images," IEEE Transactions on Geoscience and Remote Sensing, pp. 1-13, 2020.

[66] M. Papadomanolaki, S. Verma, M. Vakalopoulou, S. Gupta, and K. Karantzalos, "Detecting urban changes with recurrent neural networks from multitemporal Sentinel-2 data," in IGARSS, Yokohama, Japan, Jul. 2019.

[67] R. Pascanu, T. Mikolov, and Y. Bengio, "On the difficulty of training recurrent neural networks," in Proceedings of the 30th International Conference on International Conference on Machine Learning - Volume 28, ser. ICML'13. JMLR.org, 2013, pp. III-1310-III-1318. [Online]. Available: http://dl.acm.org/citation.cfm?id=3042817.3043083
[68] A. Paszke, S. Gross, S. Chintala, G. Chanan, E. Yang, Z. DeVito, Z. Lin, A. Desmaison, L. Antiga, and A. Lerer, "Automatic differentiation in PyTorch," in NIPS Autodiff Workshop, 2017.

[69] N. Teimouri, M. Dyrmann, and R. Jørgensen, "A novel spatio-temporal fcn-lstm network for recognizing various crop types using multitemporal radar images," Remote Sensing, vol. 11, p. 990, 042019.

[70] Z. Ebrahimzadeh, M. Zheng, S. Karakas, and S. Kleinberg, "Deep learning for multi-scale changepoint detection in multivariate time series," ArXiv, vol. abs/1905.06913, 2019.

[71] H. Lyu, H. Lu, and L. Mou, "Learning a transferable change rule from a recurrent neural network for land cover change detection," Remote. Sens., vol. 8, p. 506, 2016.

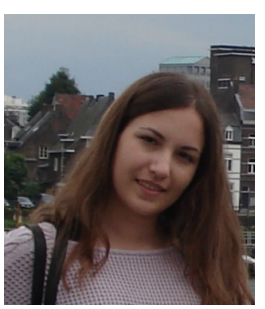

Maria Papadomanolaki Maria Papadomanolaki received the master's degree in rural and surveying engineering from the National Technical University of Athens, Greece, in 2016. She is currently pursuing the Ph.D. degree with the Remote Sensing Laboratory of the National Technical University of Athens, Greece. She is also collaborating with the MICS laboratory (Laboratoire mathématiques et informatique pour la complexité et les systémes), CentraleSupélec, France.

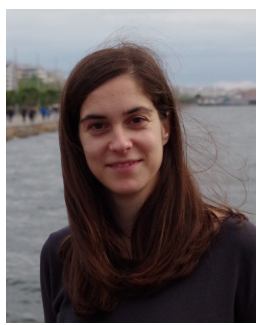

Maria Vakalopoulou M. Vakalopoulou is an assistant professor of Artificial Intelligence in the lab of Mathematics and Computer Science (since 2019), at CentraleSupélec, the school of engineering of the University Paris-Saclay. She holds an engineering diploma from National Technical University of Athens (2012) and a PhD in Computer Science and Remote Sensing (2017). Her research interests include machine and deep learning, medical imagery and remote sensing. The researcher has published her research in top-rank international journals and conferences and she has received a number of awards for her research contributions. She has served as an area chair in multiple conference and as reviewer in multiple journals and conferences in the fields of medical imaging, remote sensing and artificial intelligence.

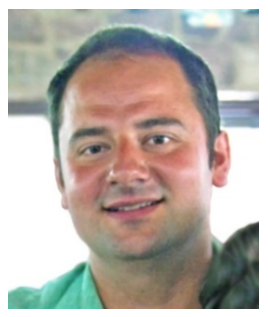

Konstantinos Karantzalos Konstantinos Karantzalos (http://users.ntua.gr/karank) received his engineering diploma from the National Technical University of Athens (NTUA, Greece) and his PhD (2007, NTUA) in collaboration with Ecole Nationale de Ponts et Chaussees (ENPC, France). In 2007, he joined the Department of Applied Mathematics at Ecole Centrale de Paris (ECP, France) as a postdoc. $\mathrm{He}$ is currently an Associate Professor of Remote Sensing at the National Technical University of Athens. His teaching and research interests include geoscience and earth observation, geospatial data analytics, spectral data analysis and machine learning with applications in e.g. environmental monitoring and precision agriculture. He has several publications in top-rank international journals \& conferences and a number of awards and honors for his research contributions. Dr. Karantzalos currently serves on the Board of Directors of the Greek Space Center. 